$\underline{\text { Preprint typeset in JHEP style - HYPER VERSION }}$

DAMTP-2003-57

hep-th/0308061

\title{
D3-branes on the Coulomb branch and instantons
}

\author{
Michael B. Green and Christian Stahn \\ Department of Applied Mathematics and Theoretical Physics \\ Wilberforce Road, Cambridge CB3 OWA, UK \\ E-mail: M.B.Green@damtp.cam.ac.uk; C.Stahn@damtp.cam.ac.uk
}

\begin{abstract}
The relative coefficients of higher derivative interactions of the IIB effective action of the form $C^{4},\left(D F_{5}\right)^{4}, F_{5}^{8}, \ldots$ (where $C$ is the Weyl tensor and $F_{5}$ is the five-form field strength) are motivated by supersymmetry arguments. It is shown that the classical supergravity solution for $N$ parallel $D 3$-branes is unaltered by this combination of terms. The non-vanishing of ${ }^{0} C^{2}$ in this background (where ${ }^{0} C$ is the background value of the Weyl tensor) leads to effective $O\left(\alpha^{\prime-1}\right)$ interactions, such as $C^{2}$ and $\Lambda^{8}$ (where $\Lambda$ is the dilatino). These contain $D$-instanton contributions in addition to tree and one-loop terms. The near horizon limit of the $N D$ 3-brane system describes a multi- $A d S_{5} \times S^{5}$ geometry that is dual to $\mathcal{N}=4 S U(N)$ Yang-Mills theory spontaneously broken to $S\left(U\left(M_{1}\right) \times \cdots \times U\left(M_{r}\right)\right.$ ). Here, the D3-branes are grouped into $r$ coincident bunches with $M_{r}$ in each group, with $\sum_{r} M_{r}=N$ and $M_{r} / N=m_{r}$ fixed as $N \rightarrow \infty$. The boundary correlation function of eight $\Lambda$ 's is constructed explicitly. The second part of the paper considers effects of a constrained instanton in this large $-N$ Yang-Mills theory by an extension of the analysis of Dorey, Hollowood and Khoze of the one-instanton measure at finite $N$. This makes precise the correspondence with the supergravity $D$-instanton measure at leading order in the $1 / N$ expansion. However, the duality between instanton-induced correlation functions in Yang-Mills theory and the dual supergravity is somewhat obscured by complications relating to the structure of constrained instantons.
\end{abstract}

KEYwords: Superstring; D-instanton; Yang-Mills instanton. 


\section{Contents}

1. Introduction

2. Type IIB effective action, $D 3$-branes and $D$-instantons

2.1 Supersymmetry and the generalised $C^{4}$ interaction 6

2.2 Non-renormalization of $D 3$ background at $O\left(\alpha^{\prime-1}\right)$

$2.3 O\left(\alpha^{\prime-1}\right)$ interactions in $D 3$ backgrounds 11

2.3.1 The dilatino propagator and the $\Lambda^{8}$ amplitude 12

2.3.2 D-instanton zero modes 13

3. Instantons in non-conformal $\mathcal{N}=4$ supersymmetric Yang-Mills 14

3.1 Review of the one-instanton measure 14

3.2 Multi-centred configurations 17

4. Summary 21

A. Properties of the D3-brane background supergravity solution 22

B. Instanton measure and Schur polynomials 25

B.1 Properties of the function $I_{N}$

B.2 Large- $N$ limit 27

\section{Introduction}

The AdS/CFT correspondence [1, 2, 3] has proved useful in pinning down a number of features of quantum string theory on the one hand and supersymmetric Yang-Mills quantum field theory on the other hand. The literature on this subject is large and it is sometimes difficult to decipher those aspects of the correspondence that follow from elementary symmetry principles and those that appear to contain some more detailed dynamical statements.

An intriguing series of identities that follows from the correspondence, even though it is not altogether clear why it should, concerns properties of Yang-Mills instantons and $D$-instantons of string theory. As observed in [4] and [5] there is an obvious qualitative correspondence between these objects, even in the $S U(2)$ Yang-Mills theory. Semi-classical expressions for the one-instanton contributions to the correlation functions of operators in the multiplet of superconformal currents in $S U(2)$ superconformal $\mathcal{N}=4$ Yang-Mills theory correspond to the dual supergravity amplitudes. However, it is only in the large- $N$ limit of $S U(N)$ Yang-Mills theory that the correspondence is exact [6]. The measure on the 
ten-dimensional bosonic instanton moduli space was there shown to be that of $A d S_{5} \times S^{5}$, which is identical to the geometric measure of a point-like $D$-instanton in IIB superstring theory in the presence of a large number of coincident D3-branes. Further evidence for this correspondence is contained in [7]. Half of the sixteen Poincaré supersymmetries and sixteen conformal supersymmetries of $\mathcal{N}=4$ Yang-Mills theory are broken by the presence of an instanton, which therefore carries fermionic moduli corresponding to eight broken Poincaré supersymmetries and eight broken conformal supersymmetries. These correspond to the moduli associated with the super-isometries of the maximally supersymmetric IIB supergravity background that are broken by the presence of a $D$-instanton.

The superconformal theory is a very special point in the moduli space of $\mathcal{N}=4$ theories, with vanishing expectation values for the scalar fields. More generally, nonzero expectation values correspond to displacing the $D 3$-branes in transverse directions. This breaks the conformal symmetry but the background still preserves half the Poincaré supersymmetries. The purpose of this paper is to explore properties of $D$-instantons in this background and to make contact with the instanton in non-superconformal domains of the $\mathcal{N}=4$ Yang-Mills theory.

To begin with, in section 2 we will enlarge on the supersymmetry constraints that determine the leading behaviour of $D$-instantons in the $\alpha^{\prime}$ expansion of the IIB effective action. It is known that $D$-instantons contribute to higher-derivative terms at order $\alpha^{\prime-1}$ (where the Einstein-Hilbert term is $O\left(\alpha^{\prime-4}\right)$ ), such as $C^{4}, C^{2} \Lambda^{8}$ and many others (where $C$ is the ten-dimensional Weyl curvature and $\Lambda$ is the complex dilatino). Each of these terms is multiplied by a function of the complex scalar field $\tau$, which is highly constrained by $S L(2, \mathbb{Z})$ invariance. To understand the effects of these interactions in the multi $D 3$-brane background we need to understand precisely how the various terms are related by supersymmetry. Of particular relevance are those interactions which depend only on $C, \tau$ and $F_{5}$ (the five-form field strength) and so may have non-zero values in the $D 3$ background. These partners of the familiar $C^{4}$ are interactions of the form $\left(D F_{5}\right)^{4}, F_{5}^{8}, C^{2}\left(D F_{5}\right)^{2}$ among others, all of which have a common dependence on $\tau$. Although we have not completed a full supersymmetry analysis we will present compelling evidence that these terms package together in a simple manner with precisely defined relative coefficients. This particular combination of terms will be shown to leave the background D3-brane supergravity solutions unaltered at $O\left(\alpha^{\prime-1}\right)$. A simple argument will demonstrate that although ${ }^{0} C^{4}$ is non-zero it cancels with the background values of the related terms so that the dilaton onepoint function vanishes ${ }^{1}$. Furthermore, the graviton and $F_{5}$ one-point functions will also be seen to vanish. However, ${ }^{0} C^{2}$ is non-vanishing and receives no corrections from terms involving ${ }^{0} F_{5}$. Some relevant properties of the $D 3$-brane supergravity background geometry are described in Appendix $\mathrm{A}$ where it is shown that ${ }^{0} C^{2}=-4 H^{-3 / 2} \partial_{y}^{2} \partial_{y}^{2} \sqrt{H}$, where $H$ is the standard harmonic function that enters the D3-brane solution and is a function of the six transverse coordinates, $y^{i}$. Substituting this expression into the higher derivative interactions leads to $O\left(\alpha^{\prime-1}\right)$ interactions of the form $C^{2}, \Lambda^{8}, G^{4}$ and many others. The $D$-instanton contributions to these interactions should be dual to corresponding instanton

\footnotetext{
${ }^{1}$ Here and in the following the background value of any field, $\Phi$, will be denoted by ${ }^{0} \Phi$.
} 
contributions to correlation functions in large- $N S U(N) \mathcal{N}=4$ Yang-Mills theory on the boundary.

The $\Lambda^{8}$ correlation function is used as an illustrative example. This is obtained from the known effective $C^{2} \Lambda^{8}$ supergravity interaction in section (2.3.1) by attaching the bulk to boundary dilatino propagator to each of the eight legs. This propagator is obtained in terms of the scalar bulk to boundary propagator by solving the Dirac equation in the multi D3 background. The same expression is obtained in section (2.3.2) by directly constructing the profile of the dilatino in the classical $D$-instanton background. This is linear in the Grassmann coordinates corresponding to the supermoduli for the broken supersymmetries.

The analysis of section 2 applies to any geometry resulting from parallel $D 3$-branes although the correspondence with $\mathcal{N}=4$ Yang-Mills theory requires $M_{r}$ coincident $D 3$ branes at transverse relative positions labelled by $r$, where $M_{r} / N=m_{r}$ is fixed in the limit $N \rightarrow \infty$. These multi-centred backgrounds [8, 9] include the special configurations of $D 3-$ branes that have been considered in the literature [10, 11] which correspond to continuous distributions of $D 3$-branes, where the density of the distribution is large in the large- $N$ limit. These distributions preserve particular subgroups of the $R$-symmetry group of the superconformal theory.

We will turn in section 3 to consider the dual $\mathcal{N}=4$ Yang-Mills theory in the situation in which the scalar fields $\varphi_{u v}^{i}$ have non-zero constant vacuum expectation values (where $i=1, \ldots, 6$, and $u, v=1, \ldots, N$ are colour indices). The most general configuration is one in which there are $6 N$ non-zero commuting values $\left\langle\varphi_{u v}^{i}\right\rangle=\varphi_{u}^{i} \delta_{u v}$. The instanton moduli space in this situation was discussed in [12] which focused on the situation with no degenerate scalar field vacuum expectation values. This analysis will be reviewed in section 3.1. Although the quantum theory is still finite, generic backgrounds of this type are not superconformal but preserve only the super-Poincaré symmetries. This implies that, in the presence of an instanton, there are four exact bosonic moduli $x_{0}^{\mu}(\mu=0,1,2,3)$, corresponding to the broken translation invariance and eight fermionic moduli, $\eta_{\alpha}^{A}$ (where $\alpha=1,2$ is a chiral $S O(3,1)$ spinor index and $A=1,2,3,4)$. In this situation the instanton is not an exact solution of the euclidean theory but only a minimum of the action with the non-exact moduli constrained to fixed values. In other words, there is a non-trivial dependence of the instanton action on the non-exact moduli, giving rise to a nontrivial measure. For small $g_{\mathrm{YM}}$ it is possible to study the effects of such constrained instantons in a systematic manner in perturbation theory [13, 14, 15].

In section 3.2 we will show that the instanton measure of [12] remains valid in cases in which there are degeneracies of vacuum expectation values. We will reexpress the measure in terms of an integral representation which is useful for discussing symmetry breaking in which the vacuum expectation values cluster into $r$ sets of degenerate values with $M_{r}$ in each set and with $\sum_{r} M_{r}=N$. In the limit $N \rightarrow \infty$ with $M_{r} / N=m_{r}$ fixed this configuration should be equivalent to the near-horizon geometry of a multi-centred configuration of $D 3-$ branes, as in [8, 9]. The measure will be expressed as a function of the six non-exact bosonic moduli, $\chi^{i}(i=1, \ldots, 6)$ which correspond to the transverse position of the $D$-instanton in the dual description (and reduce to the scale of the instanton and its position on the five-sphere in the conformal $A d S_{5} \times S^{5}$ limit). We will show that at large $N$ this measure 
is proportional to $\partial_{\chi}^{2} \partial_{\chi}^{2} \sqrt{H}$, where $H$ is the same harmonic function as the one that enters the dual supergravity background. In appendix $\mathrm{B}$ an alternative discussion is given of the properties of the measure by writing it in terms of Schur polynomials.

In addition to the measure, we would like to evaluate instanton-induced contributions to 'minimal' correlation functions of composite gauge invariant Yang-Mills operators. These correlation functions are those in which each operator soaks up at least one of the eight Poincaré supermoduli. However, there are significant complications in this case that do not arise in the superconformal case. These arise from the fact that the $R$-symmetry group is generically completely broken due to the vacuum expectation values for the scalar fields. This leads to mixing of infinite sets of single-trace operators, as well as mixing with multi-trace operators. Combined with the fact that the constrained instantons are not exact solutions this make it difficult to evaluate the correlation functions. Further comments on these issues are made at the end of section 3.2 without reaching a firm conclusion.

\section{Type IIB effective action, $D 3$-branes and $D$-instantons}

We will first consider the effects of interactions of order $\alpha^{\prime-1}$ in the presence of $D 3$-branes in type IIB superstring theory. There are many such interactions, including the well-known $C^{4}$ interaction (where $C$ is the Weyl tensor) which, in string frame, has the form [16],

$$
\frac{c_{1}}{\alpha^{\prime}} \int d^{10} x \sqrt{\operatorname{det} g} e^{-\phi / 2} f^{(0,0)}(\tau, \bar{\tau})\left(C^{4}+\cdots\right) \text {. }
$$

Here the specific index contractions have been suppressed, $c_{1}$ is a constant and $\tau=\tau_{1}+i \tau_{2}=$ $C^{(0)}+i e^{-\phi}$ is the complex scalar field (with $C^{(0)}$ being the Ramond-Ramond scalar). The fields $\tau$ and $\bar{\tau}$ parameterise the coset space $S L(2, \mathbb{R}) / O(2)$. The function $f^{(0,0)}(\tau, \bar{\tau})$ is defined by the Eisenstein series

$$
f^{(0,0)}(\tau, \bar{\tau})=\sum_{(m, n) \neq(0,0)} \frac{\tau_{2}^{3 / 2}}{|m+n \tau|^{3 / 2}}
$$

and is invariant under $S L(2, \mathbb{Z})$. The factor of $e^{-\phi / 2}$ in (2.1) is absent in the Einstein frame. The exact expression was suggested by a variety of arguments in [16] and [17] and was shown to be a consequence of full nonlinear supersymmetry in [18]. The value of $C^{4}$ in (2.1) is non-zero in the D3-brane background. The other interactions indicated by $\cdots$ in (2.1) are those that involve $F_{5}$ as well as the Weyl curvature. These are all the interactions that have non-zero values in the D3-brane backgrounds of interest to us here, in which $F_{5}$ is not constant and the Weyl tensor is non-vanishing and all other fields are trivial. We will determine the precise form of these non-vanishing background terms in the next sub-section where we will see that they all cancel.

The procedure of [18] for determining the fully nonlinear interactions was to require closure of the on-shell supersymmetry algebra order by order in $\alpha^{\prime}$ which uniquely deter-

mines the $O\left(\alpha^{\prime-1}\right)$ corrections to the action (up to trivial field redefinitions) as well as non-trivial and highly nonlinear corrections to the supersymmetry transformations. The 
$\alpha^{\prime}$ expansion of the IIB effective action has the form

$$
\alpha^{\prime 4} S=S^{(0)}+\alpha^{\prime 3} S^{(3)}+\cdots
$$

where $S^{(0)} / \alpha^{\prime 4}$ is the classical action. Invariance of the full action (2.3) under $\varepsilon^{*}$ supersymmetry is ensured only because the classical supersymmetry transformations are modified by $O\left(\alpha^{\prime 3}\right)$ corrections so that the complete supersymmetry transformation of any field $\Phi$ has the form

$$
\delta \Phi=\delta^{(0)} \Phi+\alpha^{\prime 3} \delta^{(3)} \Phi+\cdots,
$$

where the superscripts indicate the order in the $\alpha^{\prime}$ expansion. The expressions $S^{(3)}$ and $\delta^{(3)} \Phi$ are determined (up to field redefinitions) by solving the equation

$$
\delta^{(0)} S^{(3)}+\delta^{(3)} S^{(0)}=0
$$

together with the requirement that the supersymmetry algebra closes on shell. This means that

$\left[\delta_{1}^{(0)}+\alpha^{\prime 3} \delta_{1}^{(3)}, \delta_{2}^{(0)}+\alpha^{\prime 3} \delta_{2}^{(3)}\right] \Phi \sim 2 \operatorname{Im}\left(\varepsilon_{1} \Gamma^{M} \varepsilon_{2}\right) D_{M} \Phi+k\left(\varepsilon_{1}, \varepsilon_{2}\right) \frac{\delta\left(S^{(0)}+\alpha^{\prime 3} S^{(3)}\right)}{\delta \Phi}+O\left({\alpha^{\prime}}^{6}\right)$,

so that the algebra closes on the solutions of the equation of motion defined by $S^{(0)}+\alpha^{\prime 3} S^{(3)}$. The quantity $k\left(\varepsilon_{1}, \varepsilon_{2}\right)$ is bilinear in the Grassmann parameters $\varepsilon_{1}$ and $\varepsilon_{2}$ which are sixteencomponent $S O(9,1)$ spinors. The fact that supersymmetry of the effective action links terms in the $\alpha^{\prime}$ expansion is no surprise and is a vital ingredient in the derivation of the modular forms in the various interactions.

Supersymmetry requires the presence of very many other interactions with classical values that vanish in the classical $D 3$-brane background. We will later focus on terms with a factor of $C^{2}$, which include (2.1) as well as (again suppressing the index contractions),

$$
\frac{1}{\alpha^{\prime}} \int d^{10} x \sqrt{\operatorname{det} g} e^{-\phi / 2}\left(c_{2} f^{(6,-6)}(\tau, \bar{\tau}) \Lambda^{8} C^{2}+c_{3} f^{(2,-2)}(\tau, \bar{\tau}) G^{4} C^{2}+\ldots\right),
$$

where $\Lambda$ is the complex dilatino and $G$ is a complex combination of the $R \otimes R$ and $N S \otimes N S$ three-form field strengths. As with the $C^{4}$ term, only the traceless part of the curvature (the Weyl tensor) enters in (2.7). The constants $c_{1}, c_{2}, \ldots$ are easily determined by linearised supersymmetry combined with modular transformations. The modular form $f^{(w,-w)}$ transforms under $S L(2, \mathbb{Z})$ with holomorphic weight $w$ and anti-holomorphic weight $-w$, i.e.,

$$
f^{(w,-w)}(\tau, \bar{\tau}) \rightarrow\left(\frac{c \tau+d}{c \bar{\tau}+d}\right)^{\omega} f^{(w,-w)}(\tau, \bar{\tau})
$$

when $\tau \rightarrow(a \tau+b) /(c \tau+d)$ (with $a d-b c=1$ and $a, b, c, d$ integers). The modular transformations of $f^{(w,-w)}$ compensate for the $U(1)$ transformations of the fields that are induced by $S L(2, \mathbb{Z})$. We are here fixing the $O(2) \sim U(1)$ gauge so the scalar fields are restricted to the coset $S L(2, \mathbb{R}) / O(2)$. In this case the $U(1)$ charges of the fields are $q_{P}=2$, $q_{\Lambda}=3 / 2, q_{G}=1, q_{\psi}=1 / 2, q_{R}=0$ and $q_{F_{5}}=0$, where $P=i \partial \tau /\left(2 \tau_{2}\right)$ (the scalar field $\tau$ 
does not carry a specific $U(1)$ charge). The total $U(1)$ charge carried by the fields in any of the terms in (2.1) or (2.7) is $2 w$.

Explicitly, $f^{(w-w)}$ is given by the Eisenstein series,

$$
f^{(w,-w)}(\tau, \bar{\tau})=\frac{1}{2^{w}} \frac{\Gamma\left(w+\frac{3}{2}\right)}{\Gamma\left(\frac{3}{2}\right)} \sum_{(m, n) \neq(0,0)} \frac{\tau_{2}^{3 / 2}}{|m+n \tau|^{3}}\left(\frac{m+n \bar{\tau}}{m+n \tau}\right)^{w} .
$$

This has an expansion in the string coupling $g=e^{\phi}=\tau_{2}^{-1}$ that includes two power-behaved terms, which correspond to tree-level and one-loop string theory effects. In addition there is an infinite sequence of $D$-instanton and anti $D$-instanton contributions. The coefficient of each $D$-instanton $(K>0)$ contribution is of order $\tau_{2}^{w}$ while each anti $D$-instanton $(K<0)$ contribution starts with the power $\tau_{2}^{-w}$. In linearised approximation each interaction term involves a product of $p$ fluctuations of fields, $\prod_{r=1}^{M} \Phi_{r}$, where $p=w+4$ and the charge $K$ $D$-instanton contribution is proportional to

$$
g^{4-p} e^{2 \pi i K \tau}(2 \pi|K|)^{1 / 2} \sum_{m \mid K} \frac{1}{m^{2}}\left(1+O\left(\tau_{2}^{-1}\right)\right) .
$$

\subsection{Supersymmetry and the generalised $C^{4}$ interaction}

In order for the classical multi $D 3$-brane background to remain unaltered by the $O\left(\alpha^{\prime-1}\right)$ interactions it is important that the contributions of these higher derivative interactions to the one-point functions of the dilaton, graviton and $F_{5}$ all vanish. For example, the vanishing of the dilaton one point function requires the terms in the higher derivative action (2.1) to vanish in the classical background since they involve the dilaton-dependent factor $f^{(0,0)}(\tau, \bar{\tau})$ which would otherwise alter the dilaton equation of motion. Since the background Weyl tensor, ${ }^{0} C$, the background five-form field strength, ${ }^{0} F_{5}$, and ${ }^{0} D^{0} F_{5}$ are not zero in the non-conformal backgrounds of interest there must be detailed cancellations between the various terms in (2.1). We will now see that this follows from the BPS condition for the background.

The 32 components of the type IIB supersymmetry parameters form 16-dimensional complex $S O(9,1)$ spinors $\varepsilon^{a}$ and $\varepsilon^{* a}(a=1,2, \ldots, 16)$. The supersymmetry transformations of the gravitini fields in IIB supergravity have the form [19], 20]

$$
\begin{aligned}
\delta_{\varepsilon} \psi_{M} & =\left(D_{M}+\frac{i}{16 \cdot 5 !} \Gamma^{N_{1} \cdots N_{5}} F_{N_{1} \cdots N_{5}} \Gamma_{M}\right) \varepsilon+\cdots \equiv \mathcal{D}_{M} \varepsilon+\cdots, \\
\delta_{\varepsilon^{*}} \psi_{M}^{*} & =\left(D_{M}-\frac{i}{16 \cdot 5 !} \Gamma^{N_{1} \cdots N_{5}} F_{N_{1} \cdots N_{5}} \Gamma_{M}\right) \varepsilon^{*}+\cdots \equiv \mathcal{D}_{M} \varepsilon^{*}+\cdots,
\end{aligned}
$$

where $M, N, \ldots=0,1, \ldots, 9$ are ten-dimensional world indices, $\Gamma^{M}=e_{\hat{M}}^{M} \Gamma^{\hat{M}}$ and $e_{M \hat{M}}$ is the ten-dimensional frame field (and $\hat{M}=0,1, \ldots, 9$ is a tangent-space index) ${ }^{2}$. The $16 \times 16$ matrices $\Gamma^{\hat{M}}$ are projections of the $S O(9,1)$ acting on chiral spinors (see appendix $\mathrm{A}$ for conventions). The quantity $\mathcal{D}$ is defined to include the contribution of the five-form field strength. The transformations of the dilatini are

$$
\delta_{\varepsilon^{*}} \Lambda=\frac{i}{2} \Gamma^{M} P_{M} \varepsilon^{*}+\cdots, \quad \delta_{\varepsilon} \Lambda^{*}=\frac{i}{2} \Gamma^{M} P_{M}^{*} \varepsilon+\cdots .
$$

\footnotetext{
${ }^{2}$ Tangent space indices will be labelled by hatted indices in the following.
} 
The scalar field enters into the definition of $P_{M}=i \partial_{M} \tau /\left(2 \tau_{2}\right)$ and its complex conjugate $P^{*}$. $D_{M}$ includes the spin and Christoffel connections, as usual.

The dots in the above equations indicate the contributions of combinations of fields that have been suppressed since their precise form will not be relevant for us. For example, we are ignoring the three-form field strengths, $G_{M N P}$ and $G_{M N P}^{*}$, as well as various terms quadratic in fermions in the variation of the gravitini. Furthermore, the bosonic fields enter in combination with fermion bilinears that we are suppressing so that they form supercovariants (denoted $\hat{F}_{5}, \hat{P}$ and $\hat{P}^{*}$ in [19]). Such combinations transform under supersymmetry without derivatives of the parameters $\varepsilon$ and $\varepsilon^{*}$. By acting with $\mathcal{D}_{N}$ on equation (2.11) it is straightforward to derive the condition

$$
\delta_{\varepsilon}\left(\mathcal{D}_{[M} \psi_{N]}+\cdots\right)=\left(\mathcal{R}_{M N}+\cdots\right) \varepsilon
$$

(recalling that $\mathcal{D}$ is defined by (2.11)) where

$$
\begin{aligned}
\mathcal{R}_{M N}= & \frac{1}{8} R_{M N P Q} \Gamma^{P Q}-\frac{i}{16 \cdot 5 !} \Gamma^{K_{1} \cdots K_{5}} \Gamma_{[M} D_{N]} F_{K_{1} \cdots K_{5}} \\
& -\frac{1}{(16 \cdot 5 !)^{2}} \Gamma^{K_{1} \ldots K_{5}} \Gamma_{[M} \Gamma^{L_{1} \ldots L_{5}} \Gamma_{N]} F_{K_{1} \ldots K_{5}} F_{L_{1} \ldots L_{5}} .
\end{aligned}
$$

The combination $\left(\mathcal{D}_{[M} \psi_{N]}+\cdots\right)$ denotes the supercovariant combination of $\mathcal{D}_{[M} \psi_{N]}$ and cubic fermionic terms while $\left(\mathcal{R}_{M N}+\cdots\right)$ denotes the supercovariant extension of the curvature tensor (detailed definitions of these supercovariant contributions are given in section 9 of $\left.[20]^{3}\right)$.

The physical content of Type IIB supergravity is contained in a scalar superfield that is a function of $x^{M}, \theta$ and $\theta^{*}$, where the Grassmann coordinates $\theta$ and $\theta^{*}$ are sixteencomponent $S O(9,1)$ chiral spinors of the same chirality. This superfield satisfies an analytic constraint analogous to a chiral constraint, $D^{*} \Phi=0$, which means that it can be written as a function of $\theta$ and $\tilde{x}^{M}=x^{M}-\theta^{*} \Gamma^{M} \theta$ only. The components in the expansion of $\Phi$ in powers of $\theta$ are supercovariant combinations of fields and derivatives of fields, described above. Symbolically,

$$
\begin{gathered}
\Phi=\tau+\theta \Lambda+\theta^{2}(G+\cdots)+\theta^{3}(\mathcal{D} \psi+\cdots)+\theta^{4}(R+D F+F F+\cdots) \\
+\theta^{5}\left(D D \psi^{*}+\cdots\right)+\cdots+\theta^{8}(D D D D \bar{\tau}+\cdots)
\end{gathered}
$$

where dots indicate terms that complete each bracket into a supercovariant expression. In this case $\varepsilon$ supersymmetry implies $\delta_{\varepsilon} \Phi=\varepsilon \partial \Phi / \partial \theta$. If the Grassmann parameter is assigned a charge $1 / 2$ under $U(1)$ transformations all the terms in the superfield (2.15) have charge 2 apart from the first ( $\theta$-independent) term. The superfield is a nonlinear generalisation of the scalar superfield of [20] and was considered in detail in [21].

The detailed form of the term cubic in $\theta$ in (2.15) is (dropping the terms indicated by dots)

$$
\theta \Gamma^{M N P} \theta \mathcal{D}_{[M} \psi_{N]} \Gamma_{P} \theta
$$

\footnotetext{
${ }^{3}$ Equation (9.31) in 20] contain a small error in the supercovariant combination that includes $D_{[M} \psi_{N]}$.
} 
while the term quartic in $\theta$ is proportional to

$$
\theta \Gamma^{M N P} \theta \theta \Gamma_{P} \mathcal{R}_{M N} \theta=\theta \Gamma^{M N P} \theta \theta \Gamma^{Q R S} \theta \mathcal{R}_{M N P Q R S},
$$

where $^{4}$

$$
\begin{aligned}
& \mathcal{R}_{M N P Q R S}=\frac{1}{8} g_{P S} R_{M N Q R}+\frac{i}{48} D_{M} F_{N P Q R S} \\
& +\frac{1}{256} F_{M N P T U} F_{Q R S}{ }^{T U}-\frac{1}{768} F_{M N S T U} F_{Q R P}{ }^{T U}
\end{aligned}
$$

(we are ignoring the three-form field strength and fermion terms). Equation (2.13) follows, after adjusting a relative multiplicative constant, by applying $\varepsilon Q$ to (2.17) and identifying this with the $\varepsilon$ variation of $\mathcal{D}_{[M} \psi_{N]}$ in (2.16). In writing (2.18), it is assumed that $\mathcal{R}_{M N P Q R S}$ is symmetrised in the manner implied by multiplying by $\theta \Gamma^{M N P} \theta \theta \Gamma^{Q R S} \theta$. For example, it should be manifestly antisymmetric in $[M N P]$ and $[Q R S]$ as well as symmetric under interchange of $M N P$ and $Q R S$. Fierz rearrangements imply further symmetries, such as the absence of double traces. As a result, the components of $\mathcal{R}_{M N P Q R S}$ lie in the sum of the $\mathbf{1 0 5 0}^{+}$and $\mathbf{7 7 0}$ representations of $S O(9,1)^{5}$. The $\mathbf{7 7 0}$ is identified with the Weyl tensor, which is the only part of the Riemann tensor $R_{M N P Q}$ that survives in (2.18), as is well known. The $D F_{5}$ and $F_{5}^{2}$ terms contribute to the $\mathbf{1 0 5 0}^{+}$. The appropriately symmetrised ${ }^{0} \mathcal{R}$ can be written in spinor basis as

$$
{ }^{0} \mathcal{R}_{[a b c d]} \equiv \Gamma_{[a b}^{M N P} \Gamma_{c d]}^{Q R S}{ }^{0} \mathcal{R}_{M N P Q R S},
$$

where $a, b, c, d$ are 16-component chiral $S O(9,1)$ spinor labels. The antisymmetrisation of these indices follows from contraction with the Grassmann $\theta$ 's in (2.17) and immediately implies that there are $1820=1050+770$ components.

It is known that in linearised approximation the $O\left(\alpha^{\prime-1}\right)$ interactions in $S^{(3)}$ are contained in the integral of a function of $\Phi(\tilde{x}, \theta)$ over the sixteen $\theta$ 's. Furthermore, the full nonlinear supersymmetry uniquely determines the $\tau$-dependent modular forms $f^{(w,-w)}(\tau, \bar{\tau})$. These statements were combined in [18] to deduce the modular function $f^{(0,0)}$ that multiplies the $C^{4}$ interaction. Together with the analysis of the preceding paragraph this suggests that the $C$ and $F_{5}$ enter into the $O\left(\alpha^{\prime-1}\right)$ action in the combination

$$
S_{\mathcal{R}^{4}}^{(3)}=\int d^{10} \tilde{x} d^{16} \theta \operatorname{det} e f^{(0,0)}(\tau, \bar{\tau})\left(\theta \Gamma^{M N P} \theta \theta \Gamma^{Q R S} \theta \mathcal{R}_{M N P Q R S}\right)^{4} .
$$

This is of the form indicated in (2.1) but with precisely defined relative coefficients. The interaction $S_{\mathcal{R}^{4}}^{(3)}$ can be expanded as a combination of $C^{4}$ and terms involving $F_{5}$ and $D F_{5}$ (other fields being trivial). Since $\mathcal{R}$ contains a piece of order $F_{5}^{2}$ 21] there are highly nonlinear terms, such as $F_{5}^{8}$, in 2.20). We should emphasise that we have not proved that (2.20) contains all the terms involving only $C, F_{5}$ and $D F_{5}$. That would require

\footnotetext{
${ }^{4}$ This expression agrees with that deduced from a superfield approach in version 4 of 21 (apart from an overall factor of 2 in the definition of $\mathcal{R}$ ).

${ }^{5} \mathrm{See}$ [22] and [23] for a thorough discussion of group-theoretical aspects of $O\left(\alpha^{\prime-1}\right)$ terms in the type IIB effective action.
} 
a complete analysis of the constraints of nonlinear supersymmetry at $O\left(\alpha^{\prime-1}\right)$, which we have not completed. However, it is easy to argue how supersymmetry should determine the form of (2.20), including the modular function $f^{(0,0)}$, as follows. The classical supersymmetries acting on any field relate this $\mathcal{R}^{4}$ interaction to interactions of the form $(D \psi+\cdots) R^{2}\left(D^{2} \psi^{*}+\cdots\right)$, where the $\cdots$ inside the brackets again extend these terms to supercovariant derivatives. In order to determine the modular function it is necessary to consider the manner in which supersymmetry mixes $f^{(0,0)} \mathcal{R}^{4}$ with $f^{(1,-1)} \Lambda D \psi R^{3}$. This requires highly nonlinear modifications of the supersymmetry transformations, such as the ones deduced in 18 .

The superspace analysis of [21] apparently gives a different expression from (2.20), which we will not consider since it does not reproduce classical tree-level or one-loop string theory results.

\subsection{Non-renormalization of $D 3$ background at $O\left(\alpha^{\prime-1}\right)$}

We now want to understand how the interaction (2.20) affects BPS solutions of the classical supergravity. Such solutions are characterised by the condition that the supersymmetry transformations of the gravitini (2.11) vanish (we are only concerned with backgrounds for which the three-form field strengths vanish) so that

$$
D_{M} \zeta \equiv\left(\partial_{M}+\frac{1}{4} \omega_{M}^{\hat{M} \hat{N}} \Gamma_{\hat{M} \hat{N}}\right) \zeta=-\frac{i}{16 \cdot 5 !} \Gamma^{N_{1} \cdots N_{5}} F_{N_{1} \cdots N_{5}} \Gamma_{M} \zeta
$$

defines a Killing spinor $\zeta$, with a conjugate equation for $\zeta^{*}$. The vanishing of the transformations of the dilatini (2.12) is automatic if $P_{M}$ and $P_{M}^{*}$ vanish, which they do in the $D 3$-brane background (but $P_{M} \neq 0$ in the presence of a $D$-instanton, which will be important later). Applying $D_{N}$ to (2.21) and its conjugate leads to the condition $\mathcal{R}_{M N} \zeta=0$ together with its conjugate (setting fermion fields to zero).

Generally, the background will break some of the supersymmetry, even in the absence of the $D$-instanton. The $D 3$-brane backgrounds of concern to us break half the supersymmetry. In order to evaluate the Killing spinors we shall decompose the $S O(9,1)$-covariant fields into $S O(6) \times S O(3,1)$ representations. The $32 \times 32 S O(9,1)$ gamma matrices, $\hat{\Gamma}^{M}$, are written for $M=i+3(i=1, \ldots, 6)$ as

$$
\hat{\Gamma}^{i+3}=\hat{\gamma}^{i} \otimes \gamma_{5}=\left(\begin{array}{cc}
0 & \Sigma^{i} \\
\bar{\Sigma}^{i} & 0
\end{array}\right) \otimes \gamma_{5},
$$

where $\hat{\gamma}^{i}$ are the $8 \times 8 S O(6)$ gamma matrices and $\Sigma_{A B}^{i}$ and $\bar{\Sigma}^{i A B}$ are $4 \times 4$ matrices. For $M=\mu=0,1,2,3$,

$$
\hat{\Gamma}^{\mu}=I_{8} \otimes \gamma^{\mu}=I_{8} \otimes\left(\begin{array}{cc}
0 & \sigma^{\mu} \\
\bar{\sigma}^{\mu} & 0
\end{array}\right) .
$$

In our conventions, the ten-dimensional supersymmetry parameter $\varepsilon$ satisfies the chirality constraint $\hat{\Gamma}_{11} \varepsilon=-\varepsilon$. It decomposes into $S O(6) \times S O(3,1)$ chiral spinors,

$$
\varepsilon^{a}=\left(\varepsilon_{+\dot{\alpha}}^{A}, \varepsilon_{-A \alpha}\right),
$$


where \pm indicates the eigenvalue of $\gamma_{5}$.

The main features of the multi D3-brane background are discussed in appendix A where the well-known solution for the Weyl tensor and the five-form field strength is given in terms of a harmonic function in the transverse space, $H(y)=1+\sum_{r} M_{r} L^{4} /\left|y-y_{r}\right|^{4} \equiv e^{2 A}$. Substituting these into (2.21) leads to the solution for the Killing spinor 24,

$$
\zeta=H^{-1 / 8} \zeta_{+}^{0},
$$

where $\zeta_{+\dot{\alpha}}^{0 A}$ is an eight-component constant spinor. The corresponding condition on $\delta_{\zeta^{*}} \psi_{M}^{*}$ determines the solution for the Killing spinor

$$
\zeta^{*}=H^{-1 / 8} \zeta_{-}^{0 *} .
$$

In order to determine the ${ }^{0} \mathcal{R}^{4}$ interactions we will substitute the background fields into $\mathcal{R}_{M N R P Q S}$ which was defined in (2.18). First, we note that the $F_{5}^{2}$ terms do not contribute in this background. The non-vanishing components of the suitably symmetrised $\mathcal{R}$ are

$$
\begin{aligned}
& { }^{0} \mathcal{R}_{\mu \nu i \rho \sigma j}=\frac{1}{144}\left(2{ }^{0} g_{\mu[\rho}{ }^{0} g_{\sigma] \nu}-i \varepsilon_{\mu \nu \rho \sigma}\right) B_{i j}, \\
& { }^{0} \mathcal{R}_{i j k l m n}=-\frac{1}{48}\left(6 B_{i l}{ }^{0} g_{j m}{ }^{0} g_{k n}-i B_{i}^{p} \varepsilon_{j k l m n p}\right),
\end{aligned}
$$

where $B_{i j} \equiv 2 D_{i}^{(6)} D_{j}^{(6)} A=2 A_{, i j}-2 A_{, i} A_{, j}+{ }^{0} g_{i j} A_{, k} A^{, k}$ is introduced in appendix A. The terms with $\varepsilon$ tensors in these expressions come from ${ }^{0} D^{0} F_{5}$ while the remaining terms come from the Weyl tensor, $C$. These expressions satisfy duality conditions,

$$
\frac{1}{2} \varepsilon_{\mu \nu}{ }^{\tau \omega 0} \mathcal{R}_{\tau \omega i \rho \sigma j}=i^{0} \mathcal{R}_{\mu \nu i \rho \sigma j}, \quad \frac{1}{6} \varepsilon_{i j k}{ }^{p q r}{ }^{0} \mathcal{R}_{p q r l m n}=-i^{0} \mathcal{R}_{i j k l m n} .
$$

In the notation of (2.19) these conditions mean that ${ }^{0} \mathcal{R}$ only contains the part that is of definite chirality with respect to both $S O(3,1)$ and $S O(6)$, namely the part with $\gamma_{5}=+1$ and $\hat{\gamma}_{7}=-1$. This means that in $S O(3,1) \times S O(6)$ spinor notation the non-zero components are

$$
{ }^{0} \mathcal{R}_{[(A, \dot{\alpha})(B, \dot{\beta})(C, \dot{\gamma})(D, \dot{\delta})]},
$$

with no upper $S O(6)$ or undotted $S O(3,1)$ spinor indices. As expected for a $1 / 2$-BPS configuration the effective dimensionality of each of the four bi-spinor indices is eight.

Now decompose $\theta$ in terms of the $S O(6) \times S O(3,1)$ bi-spinors $\theta_{+\dot{\alpha}}^{A}$ and $\theta_{-\alpha} A$. From (2.29) it follows that the only components of $\theta$ that contribute to (2.17) are the eight $\theta_{+}$components. Therefore terms in $\theta^{16} \mathcal{R}^{4}$ with three or four powers of $\theta_{+}^{4}{ }^{0} \mathcal{R}$ vanish identically since they involve more than eight powers of $\theta_{+}$. This means, for example, that even though ${ }^{0} C^{4}$ is non-zero the combination of interactions in ${ }^{0} \mathcal{R}^{4}$ in $(2.20)$ cancel in the multi $D 3$-brane background, which implies that the dilaton equation of motion is unchanged. This cancellation between ${ }^{0} C^{4},{ }^{0} C^{2}\left({ }^{0} D^{0} F_{5}\right)^{2}$ and $\left({ }^{0} D^{0} F_{5}\right)^{4}$ terms can be seen explicitly by substituting the expressions (2.27). Similarly, ${ }^{0} \mathcal{R}^{3}$ vanishes since it involves twelve powers of $\theta_{+}$, which implies that the graviton and $F_{5}$ one-point functions vanish. We conclude that the classical $D 3$ background is unaltered by the $\mathcal{R}^{4}$ interactions. The first non-vanishing power is ${ }^{0} \mathcal{R}^{2}$, which leaves eight powers of $\theta_{-}$to be saturated by external field insertions in scattering amplitudes. This will be considered in the next subsection. 


\section{3 $O\left(\alpha^{\prime-1}\right)$ interactions in $D 3$ backgrounds}

We now wish to consider the $D$-instanton contribution to the $O\left(\alpha^{\prime-1}\right)$ interactions in the D3-brane background and compare them with the Yang-Mills instanton contribution (restricted to the one-instanton sector) to the corresponding correlation functions. In order to do this we need to substitute the background values of the fields into the interaction lagrangians such as, such as $C^{4}, \Lambda^{8} C^{2}, G G^{*} C^{2}, G^{4} C^{2}$. To leading order in the fluctuating fields this leads to interactions of the form

$$
\int d^{4} x d^{6} y\left(\operatorname{det}^{0} e\right){ }^{0} C^{2} \mathcal{O},
$$

where $\mathcal{O}$ is a term of the form $C^{2}, \Lambda^{8}, G G^{*}, G^{4}$, or one of the many other possibilities. According to the AdS/CFT correspondence the fields in $\mathcal{O}$ couple to the UV Yang-Mills theory on the boundary at $r \rightarrow \infty$.

In writing (2.30) we have used the fact that

$$
\begin{aligned}
\int d^{8} \theta_{+} & \theta_{+} \Gamma^{M_{1} N_{1} R_{1}} \theta_{+} \theta_{+} \Gamma^{M_{2} N_{2} R_{2}} \theta_{+}{ }^{0} \mathcal{R}_{M_{1} N_{1} R_{1} M_{2} N_{2} R_{2}} \\
& \theta_{+} \Gamma^{M_{3} N_{3} R_{3}} \theta_{+} \theta_{+} \Gamma^{M_{4} N_{4} R_{4}} \theta_{+}{ }^{0} \mathcal{R}_{M_{3} N_{3} R_{3} M_{4} N_{4} R_{4}}=c{ }^{0} C^{2}
\end{aligned}
$$

(where $c$ is a numerical constant) and gets no contribution from $F_{5}$ or $D F_{5}$. To see this we first note that the Grassmann integrations result in a tensor proportional to

$$
g^{M_{1} M_{2}} g^{N_{1} N_{3}} g^{R_{1} R_{4}} g^{N_{2} R_{3}} g^{R_{2} N_{4}} g^{M_{3} M_{4}}+\text { perms . }
$$

This means that each factor of ${ }^{0} \mathcal{R}$ has an internal pair of indices contracted. However, any non-trivial contraction of ${ }^{0} \mathcal{R}$ kills the ${ }^{0} D^{0} F_{5}$ term which means that any contraction of the form ${ }^{0} R^{M}{ }_{N P M R S}$ is proportional to the Weyl tensor, ${ }^{0} C_{N P R S}$. Furthermore, there is a unique non-vanishing contraction of two Weyl tensors, which is the combination occurring in (2.31). The factor of $\left(\operatorname{det}{ }^{0} e\right)^{0} C^{2}$ that appears in each of the interactions in (2.30) is evaluated in appendix $\mathrm{A}$ where it is shown that

$$
\left(\operatorname{det}{ }^{0} e\right)^{0} C^{2}=-4 H^{-1} \partial^{2} \partial^{2} H^{1 / 2},
$$

where $H=1+\sum_{r} M_{r} L^{4} /\left|y-y_{r}\right|^{4}$ is the standard harmonic function in the transverse space that enters into the $D 3$-brane metric (and $L$ is an arbitrary length scale).

The fields in the composite operators $\mathcal{O}$ multiply the remaining Grassmann variables, namely, the eight $\theta_{-}$variables. This determines the tensor structure of these combinations of fields. For example, the eight-dilatino term has the form $\prod_{r=1}^{8}\left(\theta_{-A_{r}}^{\alpha_{r}} \Lambda_{-\alpha_{r}}^{A_{r}}\right)$, where $\Lambda_{-}$ is the negative chirality component of the complex dilatino.

The preceding analysis applies to any configuration of $N$ parallel $D 3$-branes. However, in order to make contact between the $\alpha^{\prime}$ expansion of the classical string theory and YangMills theory it is necessary to consider D3-branes in the limit in which each brane is close to the horizon of all the other ones. After an appropriate rescaling of coordinates the constant term in $H$ can be dropped in this limit, as usual. 


\subsubsection{The dilatino propagator and the $\Lambda^{8}$ amplitude}

We will now consider the example of the effective $f^{(6,-6)}(\tau, \bar{\tau}) \Lambda^{8}{ }^{0} C^{2}$ interaction in (2.7) which gives rise to a correlation function of eight $\Lambda_{-}$operators on the $r \rightarrow \infty$ boundary. The tensor structure in this term is uniquely specified since the eight components of $\Lambda_{-}$ are antisymmetrised. Explicitly, $\int d^{8} \theta_{-} \prod_{r=1}^{8} \theta_{-A_{r}}^{\alpha_{r}} \Lambda_{-\alpha_{r}}^{A_{r}} \equiv\left(T_{8}\right)_{A_{1} \cdots A_{8}}^{\alpha_{1} \cdots \alpha_{8}} \prod_{r=1}^{8} \Lambda_{-\alpha_{r}}^{A_{r}}$, where the tensor $T_{8}$ is the unique singlet under $S O(6)$ and $S O(3,1)$ which would simply be the $S O(8)$ epsilon tensor, $\varepsilon^{a_{1} \cdots a_{8}}$, if the eight fermionic collective coordinates were assembled into an $S O(8)$ spinor.

The $D$-instanton contributions are obtained by considering the Fourier expansion of $f^{(6,-6)}$. From $(2.10)$ we see that to leading order in the string coupling constant the $D$ instanton contributions to $\Lambda_{-}^{8}$ are proportional to

$$
f_{K}^{(6,-6)} \sim g^{-6}(2 \pi|K|)^{1 / 2} \mu(K) e^{2 \pi i K \tau} .
$$

The correlation function of eight $\Lambda$ 's at points $x_{r}^{\mu_{r}}$ on the boundary $|y| \rightarrow \infty$ is obtained by attaching a bulk to boundary propagator to each $\Lambda_{-}$in the interaction vertex. The propagator connecting the interaction point $\left(x_{0}^{\mu}, y_{0}^{i}\right)$ to the appropriate point on the boundary is obtained by solving the ten-dimensional Dirac equation for the dilatino,

$$
\Gamma^{M} D_{M}^{(0)} \Lambda=e_{\hat{M}}^{M} \Gamma^{\hat{M}}\left(\partial_{M}^{(0)}+\frac{1}{4} \omega_{M}^{\hat{M} \hat{N}} \Gamma_{\hat{M} \hat{N}}\right) \Lambda=-\frac{i}{4 \cdot 5 !} \Gamma^{M_{1} \cdots M_{5}} F_{M_{1} \cdots M_{5}} \Lambda
$$

where the derivatives are with respect to $x_{0}^{\mu}$ and $y_{0}^{i}$. Substituting the background fields in the $D$ background results in the equation

$$
\left(\Gamma^{i} \partial_{i}^{(0)}+\Gamma^{\mu} \partial_{\mu}^{(0)}+\left(\frac{1}{4}+\frac{1}{2} \gamma^{5}\right) \Gamma^{i} A_{, i}\right) \Lambda=0,
$$

where $\gamma^{5} \Gamma^{i} A_{, i} / 2$ is the $y_{0}$-dependent 'mass term' while $\Gamma^{i} A_{, i} / 4$ comes from the spin connections. Writing $\Lambda=\Lambda_{-}+\Lambda_{+}$gives the coupled equations,

$$
\left(\hat{\gamma}^{i} \partial_{i}^{(0)}-\frac{1}{4} \hat{\gamma}^{i} A_{r}\right) \Lambda_{-}=\gamma^{\mu} \partial_{\mu}^{(0)} \Lambda_{+}
$$

and

$$
\left(\hat{\gamma}^{i} \partial_{i}^{(0)}+\frac{3}{4} \hat{\gamma}^{i} A_{i}\right) \Lambda_{+}=-\gamma^{\mu} \partial_{\mu}^{(0)} \Lambda_{-}
$$

It follows that

$$
\partial_{x_{0}}^{2} \Lambda_{-}+H^{-1}\left(\hat{\gamma}^{\hat{\imath}} \partial_{\hat{\imath}}^{(0)}-\frac{1}{4} \hat{\gamma}^{\hat{\imath}} A_{\hat{\imath}}\right)\left(\hat{\gamma}^{\hat{\jmath}} \partial_{\hat{\jmath}}^{(0)}-\frac{1}{4} \hat{\gamma}^{\hat{\jmath}} A_{\hat{\jmath}}\right) \Lambda_{-}=0
$$

where the hats again indicate flat tangent space indices. The dilatino solution therefore has the form

$$
\Lambda_{-\alpha}^{A}\left(x_{0}, y_{0}\right)=\pi^{3} \int d^{4} x \hat{K}_{\Lambda}\left(x-x_{0} ; y_{0}\right) \tilde{\Lambda}_{-\alpha}^{A}(x),
$$

where $\tilde{\Lambda}_{-}^{A}(x)$ denotes the value of the dilatino at the point $x$ of the boundary at $|y|=r \rightarrow$ $\infty$. The dilatino bulk to boundary propagator is given by

$$
\hat{K}_{\Lambda}=\hat{K}\left(x-x_{0} ; y_{0}\right) H^{1 / 8}\left(y_{0}\right),
$$


where $\hat{K}$ is the scalar bulk to boundary propagator, which satisfies the scalar Laplace equation,

$$
\left(H^{1 / 2} \partial_{x_{0}}^{2}+H^{-1 / 2} \partial_{y_{0}}^{2}\right) \hat{K}=0 .
$$

The $D$-instanton part of the eight-dilatini amplitude that emerges from the $D 3$-brane background is therefore proportional to

$$
\begin{aligned}
& g^{2} e^{-2 \pi\left(i C^{(0)}+1 / g\right)} \int d^{4} x_{0} d^{6} y_{0} d^{8} \eta\left(\partial_{y_{0}}^{2} \partial_{y_{0}}^{2} H^{1 / 2}\left(y_{0}\right)\right) \\
&\left(T_{8}\right)_{A_{1} \cdots A_{8}}^{\alpha_{1} \cdots \alpha_{8}} \prod_{r=1}^{8}\left(\frac{1}{g} \hat{K}\left(x_{r}-x_{0} ; y_{0}\right) \tilde{\Lambda}_{-\alpha_{r}}^{A_{r}}\left(x_{r}\right)\right) .
\end{aligned}
$$

The overall factor of $g^{-6}$ in (2.34) is seen from (2.43) to come from a factor of $1 / g$ for each external state and a factor of $g^{2}$ in the measure. Since $g$ is identified with $g_{\mathrm{YM}}^{2} / 4 \pi$ this implies a factor of $g_{\mathrm{YM}}^{4}$ in the Yang-Mills instanton measure in accord with [12] and the expression in section 3 .

\subsection{2 $D$-instanton zero modes}

The single $D$-instanton induced $\Lambda^{8}$ correlation function (2.43) may also be obtained by a semi-classical analysis of the fermionic zero modes of the $D$-instanton solution.

A $D$-instanton by itself breaks sixteen of the supersymmetries [25] so that when the fields are set equal to their background values only the $\varepsilon$ symmetry is preserved and the $\varepsilon^{*}$ supersymmetry is broken, resulting in sixteen fermionic moduli. This is seen from (2.12) by recalling that in a $D$-instanton background $P_{M} \neq 0$ but $P_{M}^{*}=0$ (after continuing to euclidean signature). In the $D 3$-brane background the $\varepsilon_{+}^{*}$ supersymmetries are already broken by the background and so they do not correspond to exact $D$-instanton moduli. Therefore, the net result of adding a $D$-instanton to the $D 3$-brane background is that the eight components of the $\varepsilon_{-}^{*}$ correspond to exact supermoduli since these are exact symmetries of the background that are broken by the $D$-instanton.

These supermoduli must be soaked up by the operators in any $D$-instanton induced correlation function. This means that the expectation value of $\mathcal{O}$ is obtained to leading order in the coupling by replacing the fields in $\mathcal{O}$ by their instanton profiles, or zero modes, as in the $A d S_{5} \times S^{5}$ case considered in [5]. The profiles are obtained by applying the broken supersymmetries to the $D$-instanton recursively. For example, identifying $\zeta^{*}$ with the broken supersymmetry, the dilatino zero modes following from 2.12) are

$$
\Lambda_{0} \equiv \delta \Lambda=\frac{i}{2} \Gamma^{M}{ }^{0} P_{M} \zeta_{-}^{*}
$$

The quantity ${ }^{0} P_{M}=e^{-\hat{\phi}} \partial_{M} e^{\hat{\phi}}$ is the classical value of $P_{M}$ in the $D$-instanton background and satisfies satisfies $\left({ }^{0} D^{M}+2 i^{0} Q_{M}\right){ }^{0} P_{M}=0$ where $e^{\hat{\phi}}$ is the solution of the scalar Green function between two points in the bulk, $(x, y)$ and $\left(x_{0}, y_{0}\right)$. The term $2 i^{0} Q_{M}$ arises from the fact that $P_{M}$ has $U(1)$ charge 2 and ${ }^{0} Q_{M}=i^{0} P_{M} / 2$ is the composite $U(1)$ gauge potential due to the $D$-instanton (with euclidean signature). For convenience we normalise 
the Killing spinor $\zeta^{*}$ in $(2.44)$ so that it has the form

$$
\zeta_{-}^{*}=\left(\frac{H\left(y_{0}\right)}{H(y)}\right)^{\frac{1}{8}} e^{\hat{\phi} / 4} \zeta_{-}^{0 *},
$$

where $\zeta_{-}^{0 *}$ is a constant eight-component Grassmann valued chiral spinor, which we take to have negative ten-dimensional chirality in accord with the chirality of the gravitino. The factor of $e^{\hat{\phi} / 4}$ in the Killing spinor is due to the $U(1)$ connection in the presence of the $D$-instanton [5, 25] $]^{6}$.

The expression (2.44) for $\Lambda_{0}$ is guaranteed to satisfy the Dirac equation on the coordinates $(x, y)$,

$$
\Gamma^{M} D_{M} \Lambda_{0}=\frac{1}{2} \gamma^{5} \Gamma^{i} A_{i} \Lambda_{0}
$$

The $y$-dependent mass term in this equation has the opposite sign from the mass term in (2.36) because this is the Dirac equation appropriate to $\Lambda^{*}$, which is conjugate to $\Lambda$. Furthermore, the $\left(x_{0}, y_{0}\right)$-dependent normalisation of the Killing spinor in (2.45) has been chosen so that $\Lambda_{0}$ reduces to the suitably rescaled bulk to boundary dilatino propagator in the limit $r \rightarrow \infty$. Close to the boundary the metric approaches $A d S_{5} \times S^{5}$ and $\left(e^{\hat{\phi}}-\right.$ $g) \sim r^{-4} \hat{K}$, where $\hat{K}$ is the scalar bulk to boundary propagator. Therefore, in this limit $r P_{r} \rightarrow-4 g^{-1} r^{-4} \hat{K}$. Together with the fact that $H^{-1 / 8}(r) \rightarrow r^{1 / 2}$ and $e^{\hat{\phi}} \rightarrow g$ in this limit it follows from (2.44) that

$$
\lim _{r \rightarrow \infty} \Lambda_{0}=r^{-7 / 2} g^{-3 / 4} \hat{K}\left(x-x_{0}, y_{0}\right) H^{1 / 8}\left(y_{0}\right) \zeta_{-A \alpha}^{0 *} \tilde{\Lambda}^{A \alpha}(x) \equiv \zeta_{-A \alpha}^{0 *} g^{-3 / 4} \Lambda_{-\alpha}^{A}\left(x_{0}, y_{0}\right),
$$

where $\Lambda_{-\alpha}^{A}$ is the same expression as (2.40). The eight supermoduli $\zeta_{-}^{0 *}$ are soaked up by the product of eight dilatini in the correlation function and the Grassmann integrations generate the tensor $T_{8}$ contracted into eight propagators in agreement with (2.43). The factor of $g^{-6}$ in (2.43) is also reproduced. The factor of $\partial^{2} \partial^{2} H^{1 / 2}$ in the integrand of (2.43) should be proportional to the $D$-instanton measure, which we have not evaluated directly.

\section{Instantons in non-conformal $\mathcal{N}=4$ supersymmetric Yang-Mills}

We now turn to consider the Yang-Mills dual of the above superstring description. The displaced D3-branes correspond to vacuum values for scalar fields that break the gauge symmetry from $S U(N)$ to $S\left(U\left(M_{1}\right) \times \cdots \times U\left(M_{l}\right)\right)$. We will again be interested in the limit $N \rightarrow \infty$ with $M_{r} / N=m_{r}$ fixed. Before considering the large- $N$ limit we will review the analysis of the instanton contribution by [12] of the finite $N$ case with nondegenerate scalar field vacuum expectation values.

\subsection{Review of the one-instanton measure}

With non-zero vacuum expectation values for the scalar fields the BPST instanton is not a solution of the euclidean field equations unless its scale is constrained [14. The constrained

\footnotetext{
${ }^{6}$ In $\left[5\right.$ and [16] the factor of $e^{\hat{\phi} / 4}$ was incorrectly written as $e^{-\hat{\phi} / 4}$.
} 
instanton action depends on the scale in a manner that is controllable in perturbation theory and gives rise to a nontrivial measure. In the case of the $\mathcal{N}=4$ Yang-Mills theory the measure on the supermoduli space of such constrained instantons in the presence of non-zero expectation values for scalar fields is efficiently expressed as a decoupling limit of the corresponding $D$-brane configuration. The background of $N$ parallel $D 3$-branes at transverse positions $\varphi_{u}^{i}$ can be obtained by T-duality on a six-torus from $N D$-branes with Wilson lines in the six toroidal directions. The $D$-instanton arises by T-duality from a $D 5$ brane with a world-volume in the toroidal directions. The supermoduli of the instanton are identified with the ground states of the open strings on the $D 5$-brane and the strings joining the $D 5$-brane to the different $D 9$-branes. The open-string ground states on the $D 5$-brane (the instanton) describes a $N=2$ vector supermultiplet consisting of the vector $\chi^{i}$ and eight fermionic partners, $\lambda_{A}^{\dot{\alpha}}$ and a $N=2$ hypermultiplet that is made up of the four broken Poincaré translations, $x_{0}^{\mu}$, and eight super-translations, $\eta_{\alpha}^{A}(m=1,2,3,4, A=1,2,3,4$ and $\alpha=1,2)$. The $D 9-D 5$ open-string ground states are the moduli $w_{u \dot{\alpha}}, \bar{w}^{u \dot{\alpha}}$ and their superpartners $\mu_{A u}, \bar{\mu}_{A}^{u}$ which fit into a $N=1$ hypermultiplets of the $D 5$-brane (the $D 5$ - $D 9$ system being $1 / 4$ BPS).

The partition function defined by these variables is given by

$$
\mathcal{Z}=\pi^{-6} g_{\mathrm{YM}}^{4} \int d^{8} \eta_{\alpha}^{A} d^{4} x_{0}^{\mu} \hat{\mathcal{Z}}
$$

where $\hat{\mathcal{Z}}$ is the centred partition function,

$$
\begin{aligned}
\hat{\mathcal{Z}}= & 2^{-2 N-1} \pi^{-6 N-9} \int d^{6} \chi d^{2 N} w d^{2 N} \bar{w} d^{8} \lambda d^{4 N} \mu d^{4 N} \bar{\mu} d^{3} D \exp \left[\bar{w}^{\dot{\alpha}} \tilde{\chi}^{2} w_{\dot{\alpha}}\right. \\
& \left.-i D^{c}\left(\tau^{c}\right)^{\dot{\alpha}}{ }_{\dot{\beta}} \bar{w}^{\dot{\beta}} w_{\dot{\alpha}}-2 g_{0}^{-2} D^{2}+2 \sqrt{2} i \pi \bar{\mu}^{A} \tilde{\chi}_{A B} \mu^{B}+i \pi\left(\bar{\mu}^{A} w_{\dot{\alpha}}+\bar{w}^{\dot{\alpha}} \mu^{A}\right) \lambda_{A}^{\dot{\alpha}}\right]
\end{aligned}
$$

and $D^{c}(c=1,2,3)$ is a standard auxiliary coordinate. The vacuum expectation values $\varphi_{u}^{i}$ are contained in the combination

$$
\tilde{\chi}_{u}^{i}=\chi^{i}-\varphi_{u}^{i} .
$$

The dimensional coupling constant in (3.2) is defined by $g_{0}=g_{\mathrm{YM}}^{2} \alpha^{\prime-2}$. We are interested in the decoupling limit $\alpha^{\prime} \rightarrow 0$ in which system reduces to the field theoretic Yang-Mills instantons and so we will set $g_{0} \rightarrow \infty$ from here on. In the superconformal theory the six bosonic moduli $\chi^{i}$ describe the unit vector on the five-sphere and the instanton scale size. It is now convenient, following [12], to integrate the moduli that carry a gauge index. The $\mu_{u}^{A}$ and $\bar{\mu}^{A u}$ integrals can be evaluated by completing the square of the fermionic terms, leading to

$$
\begin{aligned}
\hat{\mathcal{Z}}= & 2^{-2 N-1} \pi^{-2 N-9} \int d^{6} \chi d^{2 N} w d^{2 N} \bar{w} d^{8} \lambda d^{3} D\left(\prod_{u} \tilde{\chi}_{u}^{4}\right) \\
& \times \exp \left[-\frac{i \pi}{2 \sqrt{2}} \lambda_{\dot{\alpha} A} \bar{w}^{\dot{\alpha}}\left(\tilde{\chi}^{-1}\right)^{A B} w_{\dot{\beta}} \lambda_{B}^{\dot{\beta}}-\bar{w}^{\dot{\alpha}} \tilde{\chi}^{2} w_{\dot{\alpha}}-i D^{c}\left(\tau^{c}\right)^{\dot{\alpha}} \bar{w}^{\dot{\beta}} w_{\dot{\alpha}}-2 g_{0}^{-2} D^{2}\right],
\end{aligned}
$$

The $w_{u}^{\dot{\alpha}}$ and $\bar{w}^{\dot{\alpha} u}$ integrations can now be performed, giving

$$
\hat{\mathcal{Z}}=\frac{1}{2 \pi^{9}} \int d^{6} \chi d^{3} D d^{8} \lambda \prod_{u=1}^{N} \frac{\tilde{\chi}_{u}^{4}}{\tilde{\chi}_{u}^{4}+\left(D+\Xi_{u}\right)^{2}},
$$


where the fermion bilinear $\Xi$ is defined by

$$
\Xi_{u}^{c}=\frac{\pi}{2 \sqrt{2}}\left(\tau^{c}\right)_{\dot{\beta}}^{\dot{\alpha}} \lambda_{\dot{\alpha} A}\left(\tilde{\chi}_{u}^{-1}\right)^{A B} \lambda_{B}^{\dot{\beta}} .
$$

It should be noted that if all the vacuum values are equal so that $\tilde{\chi}_{u}^{i}=\chi^{i}$ and $\Xi_{u}=\Xi$, the fermionic terms in the integrand of $(3.6)$ can be eliminated by shifting the variable $D^{c}$. In that case there is superconformal invariance and the $\lambda$ integration causes the measure to vanish. It is useful to change variables from $\lambda_{A \dot{\alpha}}$ to $\bar{\xi}_{\dot{\alpha}}^{A}$ defined by $\lambda_{A \dot{\alpha}}=\chi_{A B} \bar{\xi}_{\dot{\alpha}}^{B}$. The measure then reduces to the usual superconformal measure $d^{8} \mu d^{8} \bar{\xi} d^{4} x d \rho / \rho^{-5}$, where $\rho=|\chi|^{-1}$.

The eight fermionic integrations pick out the term quartic in $\Xi$ in the Taylor expansion of the integrand of (3.5) in powers of $\Xi$. This term has a factor of $D^{-4}$ which makes the $d^{3} D$ integration convergent and, as shown in [12], the result of performing these integrals is that the measure can be written as an integral over the six components of $\chi^{i}$ in the form

$$
\hat{\mathcal{Z}}=-\frac{1}{16 \pi^{3}} \int d^{6} \chi \partial_{\chi}^{2} \partial_{\chi}^{2} I_{N}(\chi)
$$

where $\partial^{2}$ is the flat Laplace operator in the six-dimensional $\chi^{i}$ space. The function $I_{N}$ depends on $\chi^{i}$ via its dependence on the $N$ quantities,

$$
x_{u}=\tilde{\chi}^{2} \equiv\left(\chi-\varphi_{u}\right)^{i}\left(\chi-\varphi_{u}\right)^{i},
$$

and is given by

$$
I_{N}(\chi) \equiv I_{N}\left(x_{1}, \ldots, x_{N}\right)=\sum_{u=1 \ldots N} x_{u}^{-1} \prod_{\substack{v=1 \ldots N \\ v \neq u}} \frac{x_{v}^{2}}{x_{v}^{2}-x_{u}^{2}} .
$$

The integral in (3.7) was evaluated in 12 using Gauss' law, giving

$$
\hat{\mathcal{Z}}=\hat{\mathcal{Z}}_{f}+\hat{\mathcal{Z}}_{\infty}
$$

where $\hat{\mathcal{Z}}_{f}$ comes from surface integrals around the points $x_{u}=0\left(\chi=\varphi_{u}\right)$. In the nondegenerate case considered in [12] the strength of each of these singular contributions is one so that $\hat{\mathcal{Z}}_{f}=N$. The contribution $\hat{\mathcal{Z}}_{\infty}$ comes from the surface at $|\chi| \rightarrow \infty$, which is the small-instanton limit and corresponds to a $D$-instanton at the boundary of $A d S_{5}$. The asymptotic behaviour $I_{N} \sim k_{N}|\chi|^{-2}$ as $|\chi| \rightarrow \infty$ leads to $\hat{\mathcal{Z}}_{\infty}=-k_{N}$, so that

$$
\hat{\mathcal{Z}}=N-k_{N}
$$

where

$$
k_{N}=\frac{2 \Gamma(N+1 / 2)}{\Gamma(N) \Gamma(1 / 2)}
$$

For future reference we note that as $N \rightarrow \infty$,

$$
k_{N}=\frac{2}{\sqrt{\pi}} N^{1 / 2}\left(1-\frac{1}{8 N}+O\left(\frac{1}{N^{2}}\right)\right)
$$

as follows from Stirling's approximation. Some properties of $I_{N}$ are elucidated by expressing it in terms of Schur functions as discussed in appendix B. 


\subsection{Multi-centred configurations}

We now want to extend the analysis of [12] to configurations for which there is a superstring dual that can be studied in semi-classical approximation. This first requires us to demonstrate that the expression for the instanton measure applies to the situation in which the eigenvalues are degenerate. We will denote by $\varphi_{r}^{i}$ an eigenvalue that has degeneracy $M_{r}$ so that $\sum_{r=1}^{l} M_{r}=N$. In this situation, the centred partition function (3.5) becomes

$$
\hat{\mathcal{Z}}=\frac{1}{2 \pi^{9}} \int d^{6} \chi d^{3} D d^{8} \lambda F, \quad F=\prod_{r=1}^{l}\left[\frac{x_{r}^{2}}{x_{r}^{2}+\left(D+\Xi_{r}\right)^{2}}\right]^{M_{r}} .
$$

Instead of repeating similar steps to those of [12] it is useful to introduce the integral representation,

$$
F=\prod_{r=1}^{l} \frac{x_{r}^{2 M_{r}}}{\Gamma\left(M_{r}\right)} \int_{0}^{\infty} d y_{r} y_{r}^{M_{r}-1} \exp \left[-y_{r}\left(x_{r}^{2}+\left(D+\Xi_{r}\right)^{2}\right)\right] .
$$

In this representation it is straightforward to perform the $D^{c}$ integrations,

$$
\begin{aligned}
\int d^{3} D F=\pi^{3 / 2} \int_{0}^{\infty} & \left(\prod_{r=1}^{l} \frac{x_{r}^{2 M_{r}} y_{r}^{M_{r}-1}}{\Gamma\left(M_{r}\right)} d y_{r}\right)\left(\sum_{r} y_{r}\right)^{-3 / 2} \\
& \times \exp \left[\frac{\left(\sum_{r} y_{r} \Xi_{r}\right)^{2}}{\sum_{r} y_{r}}-\sum_{r} y_{r}\left(x_{r}^{2}+\Xi_{r}^{2}\right)\right]
\end{aligned}
$$

Performing the integrations over the eight factors of $\lambda$ picks out the term quartic in $\Xi$, which has the form

$$
\begin{aligned}
\int d^{8} \lambda d^{3} D F=\frac{\pi^{3 / 2}}{8} \int_{0}^{\infty} & \left(\prod_{r} \frac{x_{u}^{2 M_{r}} y_{r}^{M_{r}-1}}{\Gamma\left(M_{r}\right)} d y_{r}\right)\left(\sum_{r} y_{r}\right)^{-7 / 2} \exp \left[-\sum_{r} y_{r} x_{r}^{2}\right] \\
& \times \sum_{r s t u} y_{r} y_{s} y_{t} y_{u} \int d^{8} \lambda\left(\Xi_{r}-\Xi_{s}\right)^{2}\left(\Xi_{t}-\Xi_{u}\right)^{2} \\
=\frac{\pi^{3 / 2}}{8} \int_{0}^{\infty} & \left(\prod_{r} \frac{z_{r}^{M_{r}-1}}{\Gamma\left(M_{r}\right)} d z_{r}\right)\left(\sum_{r} \frac{z_{r}}{x_{r}^{2}}\right)^{-7 / 2} \exp \left[-\sum_{r} z_{r}\right] \\
& \times \sum_{r s t u} \frac{z_{r} z_{s} z_{t} z_{u}}{x_{r}^{2} x_{s}^{2} x_{t}^{2} x_{u}^{2}} \int d^{8} \lambda\left(\Xi_{r}-\Xi_{s}\right)^{2}\left(\Xi_{t}-\Xi_{u}\right)^{2},
\end{aligned}
$$

where $z_{r}=y_{r} x_{r}^{2}$. The Grassmann integrations over the eight components of $\lambda$ can be performed making use of the identity

$$
\begin{gathered}
\int d^{8} \lambda \prod_{i=1}^{4} \lambda \tau^{c_{i}} \Sigma^{i_{i}} \lambda=2^{8} \delta^{c_{1} c_{2}} \delta^{c_{3} c_{4}}\left(\delta^{i_{1} i_{2}} \delta^{i_{3} i_{4}}-\delta^{i_{1} i_{4}} \delta^{i_{2} i_{3}}-\delta^{i_{1} i_{3}} \delta^{i_{2} i_{4}}\right) \\
+ \text { permutations of } 1234
\end{gathered}
$$


(where the normalisation $\int d \theta \theta=2$ for Grassmann integration has been adopted). This can be used to show that

$$
\begin{aligned}
& \sum_{r s t u} \frac{z_{r} z_{s} z_{t} z_{u}}{x_{r}^{2} x_{s}^{2} x_{t}^{2} x_{u}^{2}} \int d^{8} \lambda\left(\Xi_{r}-\Xi_{s}\right)^{2}\left(\Xi_{t}-\Xi_{u}\right)^{2}=3 \cdot 2^{13} \pi^{4} \sum_{r s t u} \frac{z_{r} z_{s} z_{t} z_{u}}{x_{r}^{3} x_{s}^{3} x_{t}^{3} x_{u}^{3}} \\
&\left(-x_{r} x_{s}+2 x_{r} \tilde{\chi}_{s} \cdot \tilde{\chi}_{t}+5 \tilde{\chi}_{r} \cdot \tilde{\chi}_{s} \tilde{\chi}_{t} \cdot \tilde{\chi}_{u}+6 \frac{x_{t} x_{u}}{x_{r} x_{s}}\left(\tilde{\chi}_{r} \cdot \tilde{\chi}_{s}\right)^{2}-12 \frac{x_{u}}{x_{r}} \tilde{\chi}_{r} \cdot \tilde{\chi}_{s} \tilde{\chi}_{r} \cdot \tilde{\chi}_{t}\right) \\
&=-2^{9} \pi^{4}\left(\sum_{r} \frac{z_{r}}{x_{r}^{2}}\right)^{7 / 2} \partial_{\chi}^{2} \partial_{\chi}^{2}\left(\sum_{r} \frac{z_{r}}{x_{r}^{2}}\right)^{1 / 2}
\end{aligned}
$$

Hence

$$
\int d^{8} \lambda d^{3} D F=2^{9} \pi^{8} \partial_{\chi}^{2} \partial_{\chi}^{2} I_{\left\{M_{r}\right\}}
$$

where

$$
I_{\left\{M_{r}\right\}}\left(x_{1}, \ldots, x_{l}\right)=-\frac{1}{8 \pi^{7 / 2}} \int_{0}^{\infty}\left(\prod_{r} \frac{z_{r}^{M_{r}-1}}{\Gamma\left(M_{r}\right)} d z_{r}\right)\left(\sum_{r} \frac{z_{r}}{x_{r}^{2}}\right)^{1 / 2} \exp \left[-\sum_{r} z_{r}\right] .
$$

This expression can also be obtained from

$$
\begin{aligned}
I_{\left\{M_{r}\right\}}\left(x_{1}, \ldots, x_{l}\right) & =\frac{1}{2^{5} \pi^{5}} \int \frac{d^{3} D}{D^{4}} \prod_{r=1}^{l}\left(\frac{x_{r}^{2}}{x_{r}^{2}+D^{2}}\right)^{M_{r}} \\
& =\frac{1}{8 \pi^{4}} \int_{0}^{\infty} \frac{d D}{D^{2}} \prod_{r=1}^{l} \int_{0}^{\infty} d z_{r} \frac{z_{r}^{M_{r}-1}}{\Gamma\left(M_{r}\right)} \exp \left[-z_{r}\left(1+\frac{D^{2}}{x_{r}^{2}}\right)\right]
\end{aligned}
$$

by evaluating the gaussian integral over $D$ (dropping an irrelevant $\chi$-independent divergence at $D=0)$. In the non-degenerate case $\left(M_{r}=1\right)$ the first line of $(3.22)$ coincides with an expression in [12] where it was shown to be equal to $I_{N}(3.9)$, i.e., $I_{\left\{M_{r}=1\right\}} \equiv I_{N}$. Conversely, $I_{\left\{M_{r}\right\}}$ coincides with the limit

$$
I_{\left\{M_{r}\right\}}\left(x_{1}, \ldots, x_{l}\right)=I_{N}(\underbrace{x_{1}, \ldots, x_{1}}_{M_{1}}, \underbrace{x_{2}, \ldots, x_{2}}_{M_{2}}, \ldots, \underbrace{x_{l}, \ldots, x_{l}}_{M_{l}}) .
$$

The integral representation of $I_{\left\{M_{r}\right\}}$ in (3.21) is useful for estimating its behaviour in various limits.

Large-distance limit $|\chi| \equiv r \rightarrow \infty$. Consider an arbitrary configuration with $l=N$ centres, i.e., all $M_{r}=1$ (which includes degenerate cases). Writing

$$
x_{u}=\left(\chi-\varphi_{u}\right)^{2}=r^{2}\left(1+\varepsilon_{u}\right), \quad \varepsilon_{u}=-2 \frac{\chi \cdot \varphi_{u}}{r^{2}}+\frac{\varphi_{u}^{2}}{r^{2}},
$$

we have

$$
I_{N}\left(x_{1}, \ldots, x_{N}\right)=-\frac{1}{8 \pi^{7 / 2}} \frac{1}{r^{2}} \int_{0}^{\infty} d z_{1} \cdots d z_{N}\left(\sum_{u=1}^{N} \frac{z_{u}}{\left(1+\varepsilon_{u}\right)^{2}}\right)^{1 / 2} \exp \left[-\sum_{u} z_{u}\right] .
$$


The limit $r \equiv|\chi| \gg \varphi_{u}$ corresponds to $\varepsilon_{u} \rightarrow 0$. To leading order in the $\varepsilon_{u}$, the integrand is a function of $s=z_{1}+\cdots+z_{N}$ only, and we obtain

$$
\begin{aligned}
I_{N}\left(x_{1}, \ldots, x_{N}\right) & =-\frac{1}{8 \pi^{7 / 2}} \frac{1}{r^{2}} \int_{0}^{\infty} d s s^{1 / 2} e^{-s} \int_{0 \leq z_{1}+\cdots+z_{N-1} \leq s} d z_{1} \cdots d z_{N-1}+O\left(\varepsilon_{u}\right) \\
& =\left(-\frac{1}{16 \pi^{3}}\right) \frac{2}{\sqrt{\pi}} \frac{\Gamma(N+1 / 2)}{\Gamma(N)} \frac{1}{r^{2}}+O\left(\varepsilon_{u}\right)
\end{aligned}
$$

which is the same result as in 12,

$$
I_{N}\left(x_{1}, \ldots, x_{N}\right)=\left(-\frac{1}{16 \pi^{3}}\right) \frac{k_{N}}{r^{2}}+O\left(\varepsilon_{u}\right) .
$$

Behaviour of $I_{\left\{M_{r}\right\}}$ close to any of the $l$ centres. We can choose, without loss of generality, $x_{1}=r^{2} \ll x_{2}, \ldots, x_{l}$. In that case

$$
\begin{aligned}
& I_{\left\{M_{r}\right\}}\left(x_{1}, \ldots, x_{l}\right)=-\frac{1}{8 \pi^{7 / 2}} \frac{1}{r^{2}} \int_{0}^{\infty} d z_{1} \frac{z_{1}^{M_{1}-\frac{1}{2}}}{\Gamma\left(M_{1}\right)} \exp \left(-z_{1}\right) \\
& \quad \times \int_{0}^{\infty} d z_{2} \cdots d z_{l} \frac{z_{2}^{M_{2}-1} \cdots z_{l}^{M_{l}-1}}{\Gamma\left(M_{2}\right) \cdots \Gamma\left(M_{l}\right)}\left(1+\sum_{r=2}^{l} \frac{z_{r}}{z_{1}} \frac{r^{4}}{x_{r}^{2}}\right)^{1 / 2} \exp \left[-\sum_{r=2}^{l} z_{u}\right] .
\end{aligned}
$$

To zeroth order in $r^{2} / x_{r}$, the integrals over $z_{2}, \ldots, z_{l}$ give unity, and

$$
\begin{aligned}
I_{\left\{M_{r}\right\}}\left(x_{1}, \ldots, x_{l}\right) & =\left(-\frac{1}{16 \pi^{3}}\right) \frac{2}{\sqrt{\pi}} \frac{\Gamma\left(M_{1}+1 / 2\right)}{\Gamma\left(M_{1}\right)} \frac{1}{r^{2}}+O\left(r^{4} / x_{r}^{2}\right) \\
& =\left(-\frac{1}{16 \pi^{3}}\right) \frac{k_{M_{1}}}{r^{2}}+O\left(r^{4} / x_{r}^{2}\right)
\end{aligned}
$$

Using this as an estimate for $I$ close to each of the centres allows the explicit computation of the centred partition function (3.7),

$$
\hat{\mathcal{Z}}=\hat{\mathcal{Z}}_{f}+\hat{\mathcal{Z}}_{\infty}=\sum_{r=1}^{l} k_{M_{r}}-k_{N},
$$

where the last term comes from the integral around the point at $r=\infty$. This is a small extension of the result of [12].

Large- $N$ limit with fixed $M_{r} / N$. We will now discuss the large- $N$ limit of $I$ and $\hat{\mathcal{Z}}$ in order to see how these results fit in with the known behaviour of $D$-instanton effects in type IIB supergravity. Consider multi-centred configurations with large number of VEVs at each centre such that $M_{r}=m_{r} N$ with all $m_{r}>0$ fixed and satisfying $\sum_{r} m_{r}=1$. This leads to an integral that can be evaluated using the saddle-point method,

$$
\begin{aligned}
& I_{\left\{M_{r}\right\}}\left(x_{1}, \ldots, x_{l}\right)= \\
& \quad-\frac{1}{8 \pi^{7 / 2}} \int_{0}^{\infty}\left(\prod_{r} \frac{d z_{r}}{\Gamma\left(M_{r}\right)}\right)\left(\sum_{r} \frac{z_{r}}{x_{r}^{2}}\right)^{1 / 2} \exp \left[\sum_{r}\left(-z_{r}+\left(M_{r}-1\right) \log z_{r}\right)\right] .
\end{aligned}
$$


The exponent becomes stationary at $z_{r}=M_{r}-1$. Changing variables to $\hat{z}_{r}=z_{r}-M_{r}-1$ gives

$$
\begin{aligned}
I_{N}=-\frac{1}{8 \pi^{7 / 2}} \prod_{r} \int_{1-M_{r}}^{\infty} \frac{d \hat{z}_{r}}{\Gamma\left(M_{r}\right)}\left(\sum_{r} \frac{M_{r}-1+\hat{z}_{r}}{x_{r}^{2}}\right)^{1 / 2} \\
\quad \times\left(M_{r}-1\right)^{M_{r}-1} e^{1-M_{r}} \exp \left[-\sum_{r} \frac{\hat{z}_{r}^{2}}{2\left(M_{r}-1\right)}\right] \\
\stackrel{N \rightarrow \infty}{\longrightarrow}\left(-\frac{1}{16 \pi^{3}}\right) \frac{2}{\sqrt{\pi}}\left(\sum_{r} \frac{M_{r}}{x_{r}^{2}}\right)^{1 / 2}=-\frac{1}{8 \pi^{3} \sqrt{\pi}}\left(\sum_{r} \frac{M_{r}}{\left|\chi-\varphi_{r}\right|^{4}}\right)^{1 / 2},
\end{aligned}
$$

where Stirling's approximation has been used. We therefore see that $I_{N}$ is proportional to $\sqrt{H}$, where $H$ is a harmonic function in the six-dimensional space spanned by $\chi^{i}$. It is of course no accident that this is the harmonic function that enters into the metric for $N$ $D 3$-branes (which is reviewed in appendix A) in the near-horizon large- $N$ limit.

The scale size of the instanton is defined by $\rho^{2}=\bar{w}^{u \dot{\alpha}} w_{u \dot{\alpha}}$. Evaluating its expectation value with the measure defined by (3.2) results in $\rho^{2}=\langle\bar{w} w\rangle=2 \sum_{r} M_{r} / x_{r}$. This reduces to $\rho^{2}=2 N / r^{2}$ in the $|r| \rightarrow \infty$ (small instanton) limit and approaches $\infty$ in the various infra-red limits at $\tilde{\chi}_{r} \sim 0$ where the instanton should reduce to a BPST instanton of the $S U\left(M_{r}\right)$ subgroup. This behaviour is in qualitative agreement with expectations.

When the degeneracy of eigenvalues is finite at a number of sites the large- $N$ limit of $I_{N}$ is not equal to $\sqrt{H}$. However, as shown at the end of appendix B, the asymptotic behaviour of $I_{N}$ as a function of $r$ matches that of $\sqrt{H}$ at least up to terms of order $r^{-9}$. The derivation relies on writing $I_{N}$ in terms of Schur polynomials although this result can presumably also be extracted from the integral representation (3.9).

\section{Comments on correlation functions in $\mathcal{N}=4$ Yang-Mills theory}

The matching of correlation functions in the Yang-Mills theory with the supergravity amplitudes is not so straightforward. Even in the absence of the instanton the D3 background metric is complicated to describe in terms of the boundary Yang-Mills theory. As described in [8] and [9] the classical scalar field expectation values generically break the $S O(6) R$-symmetry, leading to an infinite tower of non-vanishing single-trace chiral primary operators, $\mathcal{O}_{l}^{\left(i_{1} \ldots i_{l}\right)}=\operatorname{Tr}\left(\varphi^{\left(i_{1}\right.} \ldots \varphi^{\left.i_{l}\right)}\right)$ as well as muti-trace products of these operators, which mix with each other. The tensor indices in these expressions are defined by the values of the scalar field expectation values. These operators couple to the trace of $h_{M N}$ on the five-sphere, where $h_{M N}=g_{M N}-g_{A d S}$ is the deviation of the metric from the $A d S_{5} \times S^{5}$ metric, $g_{A d S}$. The multi-trace components are essential for generating terms nonlinear in $M_{r}$ in the expansion of $H^{1 / 2}$ in powers of $1 / r$.

In addition, even in the superconformal theory, where the scalar fields $\varphi^{i}$ have zero classical expectation values, the presence of an instanton leads to expectation values which

are proportional to products of two fermion moduli, $\varphi_{u v}^{[A B]} \sim \bar{\mu}_{u}^{A} \mu_{v}^{B}$ (where $u$ and $v$ are $S U(N)$ indices taking $N$ values). Substituting in the expression for the chiral primary operators and integrating over these fermions gives the multi-trace condensate corresponding 
to the string-frame metric for an instanton in $A d S_{5} \times S^{5}$. Similar condensates arise for superconformal chiral descendents for which the relevant single trace operators are given by expressions such as $\mathcal{C}_{l}^{\left(i_{1} \ldots i_{l}\right)}=\operatorname{Tr}\left(\left({ }^{0} F^{-}\right)^{2} \varphi^{\left(i_{1}\right.} \ldots \varphi^{\left.i_{l}\right)}\right), \hat{\Lambda}_{l}^{A i_{1} \ldots i_{l}}=\operatorname{Tr}\left(\sigma^{\mu \nu}{ }^{0} F^{-} \lambda^{A} \varphi^{i_{1}} \ldots \varphi^{i_{l}}\right)$, etc. (where the appropriate symmetrisations of indices is assumed). In the superconformal theory $l$ would indicate the Kaluza-Klein mode on the five-sphere to which the operator couples.

When $\varphi$ has both a classical expectation value and and an instanton-induced fermion bilinear the situation is even more complicated. This has not been analysed in detail but certain qualitative features are apparent. The combination of operators that couple to the dilaton $\mathcal{C}$ in this background is a sum of single-trace operators $\mathcal{C}_{l}$ multiplied by factors of $\mathcal{O}_{l_{r}}$. In order to match the supergravity expression for the $D$-instanton solution of the dilaton this combination has to be equal to $\hat{K}$, the solution of the ten-dimensional Laplace equation (2.42). Similarly, the dilatino couples to ${ }^{0} \hat{\Lambda}$ which is a sum of single-trace operators $\hat{\Lambda}_{l}$ multiplied by factors of $\mathcal{O}_{l_{r}}$. As usual, the instanton profile of $\hat{\Lambda}_{l}$ is linear in the fermionic collective coordinates for the broken supersymmetries, $\eta_{\alpha}^{A}$. This should lead to a non-zero correlation function of eight $\hat{\Lambda}$ 's that matches the eight-dilatini correlation function (2.43).

\section{Summary}

In this paper we considered aspects of higher derivative interactions of type IIB superstring theory in the background of a collection of parallel D3-branes. To begin with we considered the higher derivative interactions of the IIB effective action at $O\left(\alpha^{\prime-1}\right)$ that are functions only of $C, \tau$ and $F_{5}$ (as well as the metric) and which might therefore be non-zero in the $D 3$ background. An argument that combined supersymmetry and $S L(2, \mathbb{Z})$ invariance was used to package all these terms into a highly nonlinear expression of the form $\alpha^{\prime-1} \int d^{10} x \operatorname{det} e f^{(0,0)} \mathcal{R}^{4}$. A full proof that there are no additional terms involving only these fields has not been completed. We saw that $\mathcal{R}$ possesses an elegant self-duality property in the $D 3$ background from which it follows that $\left({ }^{0} \mathcal{R}\right)^{4}=\left({ }^{0} \mathcal{R}\right)^{3}=0$ and so the background is not affected by the order $\alpha^{\prime-1}$ interactions. More precisely, the one-point functions of the dilaton, graviton and five-form field strength all vanish, which is in accord with stringy intuition. The non-zero value of the curvature leads to a non-zero value of $\sqrt{{ }^{0} g}{ }^{0} \mathcal{R}^{2} \sim \sqrt{{ }^{{ }_{g}}}{ }^{0} C^{2}$ proportional to $H^{-1} \partial_{y}^{2} \partial_{y}^{2} H^{1 / 2}$, where $H$ is the harmonic function that enters in the classical background solution. There are no terms quadratic in the five-form background field or its derivative. As a result, there are terms in the effective action of the form $C^{2}, \Lambda^{8}, G^{4}$ and many others, which all have known $D$-instanton contributions. The $D$-instanton contribution to the correlation function of eight $\Lambda$ 's on the $|y| \rightarrow \infty$ boundary of the near-horizon geometry was explicitly determined. This involved constructing the bulk to boundary propagator for $\Lambda_{-}$, which is given in terms of the product of a Killing spinor and the scalar bulk to boundary propagator, $\hat{K}$. This is also the structure obtained from the fermionic zero modes in the $D$-instanton background. Special solutions of the scalar Laplace equation have been discussed in the literature for continuous distributions of $D 3$-branes that have some residual symmetry (see, for example, [8, 10, 11]). 
The second part of the paper (section 3) considered the effects of an instanton in nonconformal regions of the moduli space of $\mathcal{N}=4$ supersymmetric $S U(N)$ Yang-Mills theory at large $N$. We argued that the expression for the measure of the constrained one instanton moduli space of [12] applies to degenerate cases in which eigenvalues of the scalar fields coincide. In the limit $N \rightarrow \infty$ with $M_{r} / N$ fixed (where $M_{r}$ is the degeneracy of eigenvalues with value $\varphi_{r}$ ) the measure on the six scalar moduli, $\chi^{i}$, was found to be proportional to $\partial_{\chi}^{2} \partial_{\chi}^{2} H^{1 / 2}$. This is the same factor as appeared in the $D$-instanton measure (with $y^{i}$ identified with $\chi^{i}$, apart from a dimensional constant).

The comparison of instanton induced correlation functions of gauge invariant operators with corresponding supergravity amplitudes is more problematical. The background geometry is described by a complicated sum of multi-trace operators of the boundary theory, involving the classical vacuum values of the scalar fields. The presence of the instanton induces additional expectation values of the Yang-Mills scalar fields that are bilinear in the infinite number of non-exact fermionic moduli. We have not sorted out the full effect of these vacuum values but expect that the correlation functions should match those of the supergravity.

The effective IIB supergravity action contains a great deal of information concerning multiply charged $D$-instanton contributions. This should provide information about multi Yang-Mills instantons which we have not considered explicitly in these backgrounds. The agreement between the two sides indicates, for example, that the Yang-Mills measure should contain a factor of $\sum_{m \mid K} 1 / m^{2}$, just as in the $A d S_{5} \times S^{5}$ case.

\section{Acknowledgments}

We are very grateful to Gary Gibbons, Robert Helling, Stefano Kovacs, Hugh Osborn, Malcolm Perry and Paul Townsend for useful discussions. CS would like to thank the Studienstiftung des deutschen Volkes for financial support.

\section{A. Properties of the D3-brane background supergravity solution}

We will here review some useful properties of the supergravity background considered in the main text and also define notation and conventions.

Type IIB supergravity admits multi-centre D3-brane solutions 24] with a metric of the form

$$
d s^{2}={ }^{0} g_{M N} d x^{M} d x^{N}=H^{-1 / 2} \eta_{\mu \nu} d x^{\mu} d x^{\nu}+H^{1 / 2} \delta_{i j} d y^{i} d y^{j},
$$

where $\eta_{\mu \nu}=\operatorname{diag}(-1,+1,+1,+1)$ and the indices take values $M=0, \ldots, 9, \mu=0, \ldots, 3$, $i=1, \ldots, 6$. The self-dual five-form field strength is given by

$$
\begin{aligned}
{ }^{0} F_{5} & =(1+*) d c^{(4)}, \\
c^{(4)} & =H^{-1} d x^{0} \wedge d x^{1} \wedge d x^{2} \wedge d x^{3},
\end{aligned}
$$

the dilaton is constant, and all other fields are set to zero. The field equations

$$
{ }^{0} R^{M N}-\frac{1}{2}{ }^{0} g^{M N}{ }^{0} R=\frac{1}{16 \cdot 6}{ }^{0} F^{M M_{2} \cdots M_{5}}{ }^{0} F^{N}{ }_{M_{2} \cdots M_{5}}
$$


and $d^{0} F_{5}=0$ are satisfied if $H$ is a harmonic function of the transverse coordinates $y^{i}$,

$$
H^{1 / 2} D^{M} D_{M} H(y)=\delta^{i j} \partial_{i} \partial_{j} H(y)=4 \pi^{3} L^{4} \sum_{r} M_{r} \delta^{(6)}\left(y^{i}-y_{r}^{i}\right)
$$

where $L$ is a length scale and $M_{r}$ are integers. The source terms represent a superposition of parallel $D 3$-branes, with $M_{r}$ coincident branes at $y=y_{r}$. The solution

$$
H=1+\sum_{r} \frac{L^{4} M_{r}}{\left|y-y_{r}\right|^{4}} \equiv e^{2 A},
$$

where $\left|y-y_{r}\right|^{2}=\left(y^{i}-y_{r}^{i}\right)^{2}$, leads to a metric which is asymptotically flat. The harmonic condition implies $A_{, i}{ }^{i}=-2 A_{, i} A^{i}+$ ( $\delta$ function terms).

The non-zero components of the Christoffel connection for the metric (A.1) are

$$
{ }^{0} \Gamma_{\nu i}^{\mu}=-\frac{1}{2} \delta_{\nu}^{\mu} A_{, i}, \quad{ }^{0} \Gamma_{\mu \nu}^{i}=\frac{1}{2}{ }^{0} g_{\mu \nu} A^{, i}, \quad{ }^{0} \Gamma_{j k}^{i}=\frac{1}{2}\left(\delta_{j}^{i} A_{, k}+\delta_{k}^{i} A_{, j}-{ }^{0} g_{j k} A^{, i}\right),
$$

and the Riemann tensor has non-zero components

$$
\begin{aligned}
{ }^{0} R_{\mu \nu \rho \sigma} & =-\frac{1}{2} A_{, i} A^{, i}{ }^{0} g_{\mu[\rho}{ }^{0} g_{\sigma] \nu}, \\
{ }^{0} R_{\mu i \nu j} & =\frac{1}{4}{ }^{0} g_{\mu \nu}\left(-3 A_{, i} A_{, j}+2 A_{, i j}+{ }^{0} g_{i j} A_{, k} A^{, k}\right), \\
{ }^{0} R_{i j k l} & =\left({ }^{0} g_{i[l} A_{, k] j}-\frac{1}{2}{ }^{0} g_{i[l} A_{, k]} A_{, j}+\frac{1}{4}{ }^{0} g_{i[l}{ }^{0} g_{k] j} A_{, m} A^{, m}\right)-(i \leftrightarrow j),
\end{aligned}
$$

where antisymmetrisations are with unit weight. The nonvanishing components of the Ricci tensor are

$$
{ }^{0} R_{\mu \nu}=-{ }^{0} g_{\mu \nu} A_{, i} A^{, i}, \quad{ }^{0} R_{i j}={ }^{0} g_{i j} A_{, k} A^{, k}-2 A_{, i} A_{, j},
$$

and the the curvature scalar vanishes,

$$
{ }^{0} R=0
$$

The Weyl tensor has components

$$
\begin{aligned}
{ }^{0} C_{\mu i \nu j} & =\frac{1}{4}{ }^{0} g_{\mu \nu} B_{i j}, \\
{ }^{0} C_{i j k l} & =\frac{1}{2}\left(-{ }^{0} g_{i[k} B_{l] j}+{ }^{0} g_{j[k} B_{l] i}\right) .
\end{aligned}
$$

Here we have introduced the symmetric traceless tensor

$$
B_{i j} \equiv 2 D_{i}^{(6)} D_{j}^{(6)} A=2 A_{, i j}-2 A_{, i} A_{, j}+{ }^{0} g_{i j} A_{, k} A^{, k},
$$

where $D_{i}^{(6)}$ signifies the covariant derivative with respect to the six-dimensional transverse space. The only quadratic diffeomorphism invariant that can be constructed from the Weyl tensor is

$$
{ }^{0} C_{M N P Q}{ }^{0} C^{M N P Q}=2 \operatorname{tr} B^{2}=-4 H^{-3 / 2} \partial^{2} \partial^{2} H^{1 / 2} .
$$

The field strength $F_{5}$ in (A.2) is self-dual by construction. We follow the convention where the Hodge dual is defined by

$$
(* F)_{M_{1} \cdots M_{5}}=\frac{1}{5 !} \varepsilon_{N_{1} \cdots N_{5} M_{1} \cdots M_{5}} F^{N_{1} \cdots N_{5}},
$$


and $\varepsilon_{M_{1} \cdots M_{10}}$ are the components of the volume form, i.e., $\varepsilon_{0 \cdots 9}=H^{1 / 2}$. The non-vanishing components of ${ }^{0} F_{5}$ are

$$
{ }^{0} F_{\mu \nu \rho \sigma i}=-2 \varepsilon_{\mu \nu \rho \sigma} A_{, i}, \quad{ }^{0} F_{j k l m n}=2 \varepsilon_{i j k l m n} A^{, i},
$$

where $\varepsilon_{\mu \nu \rho \sigma}$ and $\varepsilon_{i j k l m n}$ denote the components of the four- and six-dimensional volume forms, $\varepsilon_{0123}=H^{-1}$ and $\varepsilon_{123456}=H^{3 / 2}$. The covariant derivative of $F_{5}$ has components

$$
\begin{aligned}
{ }^{0} D_{i}{ }^{0} F_{j \mu \nu \rho \sigma} & =-\varepsilon_{\mu \nu \rho \sigma} B_{i j} \\
{ }^{0} D_{i}{ }^{0} F_{j k l m n} & =\left(2 A_{, i}{ }^{p}-A_{, i} A^{, p}\right) \varepsilon_{p j k l m n}+5 A^{, p} A_{[, j} \varepsilon_{k l m n] i p} .
\end{aligned}
$$

We introduce an orthonormal frame

$$
e_{\mu}^{\hat{\mu}}=H^{-1 / 4} \delta_{\mu}^{\hat{\mu}}, \quad e_{i}^{\hat{\imath}}=H^{1 / 4} \delta_{i}^{\hat{\imath}}
$$

where hats denote flat indices. The connection one-form then has components

$$
\omega_{\mu}^{\hat{\nu} \hat{\jmath}}=-\frac{1}{2} \delta_{\mu}^{\hat{\nu}} \delta_{j}^{\hat{\jmath}} A^{, j}, \quad \omega_{i}^{\hat{\jmath} \hat{k}}=\frac{1}{2}\left(\delta_{i}^{\hat{\jmath}} \delta^{\hat{k} l}-\delta_{i}^{\hat{k}} \delta^{\hat{\jmath} l}\right) A_{, l} .
$$

The $32 \times 32$ Dirac matrices $\hat{\Gamma}_{\hat{M}}$ satisfy

$$
\left\{\hat{\Gamma}_{\hat{M}}, \hat{\Gamma}_{\hat{N}}\right\}=2 \eta_{\hat{M} \hat{N}}, \quad \eta=\operatorname{diag}(-1,+1, \ldots,+1) .
$$

The chirality of a ten-dimensional spinor is defined by the eigenvalue of $\hat{\Gamma}_{11}=\hat{\Gamma}^{\hat{0}} \cdots \hat{\Gamma}^{\hat{g}}$, which has $\hat{\Gamma}_{11}^{2}=1$. The matrices $\hat{\Gamma}$ can be expressed as

$$
\hat{\Gamma}_{\hat{M}}=\left(\begin{array}{cc}
0 & \Gamma_{\hat{M}} \\
\bar{\Gamma}_{\hat{M}} & 0
\end{array}\right),
$$

where $\Gamma_{\hat{M} a b}$ and $\bar{\Gamma}_{\hat{M}}^{a b}$ are $16 \times 16$ matrices which act on chiral spinors (with upper and lower indices corresponding to \pm chiralities). A Gamma matrix with a curved index is obtained using the frame field, $\Gamma_{M}=e_{M}^{\hat{M}} \Gamma_{\hat{M}}$. In these conventions, expressions such as $\lambda_{1}^{*} \Gamma^{M_{1} \cdots M_{r}} \lambda_{2}$ transform as $S O(9,1)$ tensors. If the chiralities of $\lambda_{1}$ and $\lambda_{2}$ are equal $r$ must be odd while if the chiralities are unequal $r$ must be even. The quantity $\Gamma^{M_{1} \cdots M_{r}}$ is defined so that when when the $M_{i}$ are all distinct, it is equal to $\Gamma^{M_{1}} \bar{\Gamma}^{M_{2}} \cdots \Gamma^{M_{r}}$ if $r$ is odd and $\Gamma^{M_{1}} \bar{\Gamma}^{M_{2}} \cdots \bar{\Gamma}^{M_{r}}$ if $r$ is even (with a corresponding definition of $\bar{\Gamma}^{M_{1} \cdots M_{r}}$ ). For convenience the bars are omitted from the $\Gamma$ 's in the text since their positions are always obvious by context.

The covariant derivative acting on a spinor $\varepsilon$ is

$$
D_{\mu} \varepsilon=\partial_{\mu} \varepsilon-\frac{1}{4} A^{, j} \Gamma_{\mu j} \varepsilon, \quad D_{i} \varepsilon=\partial_{i} \varepsilon+\frac{1}{4} A^{, j} \Gamma_{i j} \varepsilon .
$$

The equations of motion of type IIB supergravity are invariant under 32 supersymmetries which form two Majorana-Weyl spinors $\varepsilon_{1}, \varepsilon_{2}$ of the same ten-dimensional chirality. The chirality is linked to the choice of sign in $F_{5}= \pm * F_{5}$, and in our conventions $\Gamma_{11} \varepsilon_{1,2}=-\varepsilon_{1,2}$. We use the complex combinations $\varepsilon=\varepsilon_{1}+i \varepsilon_{2}, \varepsilon^{*}=\varepsilon_{1}-i \varepsilon_{2}$. In the $D 3$ background the supersymmetry variation of the gravitino is

$$
\delta_{\varepsilon} \psi_{M}=D_{M} \varepsilon+\frac{i}{16 \cdot 5 !} \Gamma^{N_{1} \cdots N_{5}}{ }^{0} F_{N_{1} \cdots N_{5}} \Gamma_{M} \varepsilon=D_{M} \varepsilon-\frac{1}{4} \Gamma^{i} A_{, i} \Gamma^{(5)} \Gamma_{M} \varepsilon,
$$


with a corresponding equation for the variation of $\psi_{M}^{*}\left(\right.$ with $\left.\Gamma^{(5)}=i \Gamma^{\hat{0} \hat{1} \hat{2} \hat{3}}=\gamma^{5} \otimes 1\right)$. The background preserves those supersymmetries with $\delta_{\zeta} \psi_{M}=0$ and $\delta_{\zeta^{*}} \psi_{M}^{*}=0$. In terms of the projected Killing spinors $\zeta_{ \pm}=\frac{1}{2}\left(1 \pm \Gamma^{(5)}\right) \zeta$, this is equivalent to the conditions

$$
\begin{aligned}
\partial_{\mu} \zeta_{+} & =0, & \left(\partial_{i}+\frac{1}{2} A^{, j} \Gamma_{i j}-\frac{1}{4} A_{, i}\right) \zeta_{+} & =0 \\
\partial_{\mu} \zeta_{-} & =\frac{1}{2} \Gamma^{j} A_{, j} \Gamma_{\mu} \zeta_{+}, & \left(\partial_{i}+\frac{1}{4} A_{, i}\right) \zeta_{-} & =0 .
\end{aligned}
$$

For generic distributions of parallel $D 3$-branes the solution of these conditions is given by the 16 Killing spinors

$$
\begin{array}{ll}
\zeta_{-}=0, & \zeta_{+}=H^{-1 / 8} \zeta_{+}^{0} \\
\zeta_{+}^{*}=0, & \zeta_{-}^{*}=H^{-1 / 8} \zeta_{-}^{0 *},
\end{array}
$$

where $\zeta_{+}^{0}$ and $\zeta_{-}^{0 *}$ are constant eight-component spinors.

\section{B. Instanton measure and Schur polynomials}

In the following, we will present an alternative discussion of some of the properties of the measure of section 3.2, starting from

$$
\begin{aligned}
\hat{\mathcal{Z}} & =-\frac{1}{16 \pi^{3}} \int d^{6} \chi \partial_{\chi}^{2} \partial_{\chi}^{2} I_{N}, \\
I_{N}\left(x_{1}, \ldots, x_{N}\right) & =\sum_{u=1 \ldots N} x_{u}^{-1} \prod_{\substack{v=1 \ldots N \\
v \neq u}} \frac{x_{v}^{2}}{x_{v}^{2}-x_{u}^{2}} .
\end{aligned}
$$

In order to consider degenerate configurations of vacuum expectation values, we will need to study the function $I_{N}\left(x_{1}, \ldots, x_{N}\right)$ with its arguments set equal in clusters. Similarly, a large-distance asymptotic expansion is equivalent to taking all arguments of $I_{N}$ close to one. Both limits can be analysed by rewriting $I_{N}$ in terms of Schur polynomials, which avoids the apparent singularities at $x_{u}=x_{v}$.

\section{B.1 Properties of the function $I_{N}$}

It will prove useful to consider generalisations of $I_{N}$ defined by

$$
I_{N}^{(d)}\left(x_{1}, \ldots, x_{N}\right)=\sum_{u=1 \ldots N} x_{u}^{d} \prod_{\substack{v=1 \ldots N \\ v \neq u}} \frac{x_{v}^{2}}{x_{v}^{2}-x_{u}^{2}},
$$

where $d$ is an arbitrary integer. The $I_{N}^{(d)}$ are symmetric homogenous rational functions. A series of manipulations shows that they have particularly simple representations in terms of Schur polynomials,

$$
\begin{aligned}
I_{N}^{(d)} & =\frac{1}{\prod_{w<t}\left(x_{w}^{2}-x_{t}^{2}\right)} \sum_{u} x_{u}^{d} \prod_{v \neq u} x_{v}^{2} \frac{\prod_{w<t}\left(x_{w}^{2}-x_{t}^{2}\right)}{\prod_{v \neq u}\left(x_{v}^{2}-x_{u}^{2}\right)} \\
& =\frac{1}{\prod_{w<t}\left(x_{w}+x_{t}\right)} \frac{1}{\Delta} \sum_{u}(-1)^{N-u} x_{u}^{d} \prod_{v \neq u} x_{v}^{2} \prod_{\substack{w<t \\
w, t \neq u}}\left(x_{w}^{2}-x_{t}^{2}\right)
\end{aligned}
$$


with $\Delta=\prod_{w<t}\left(x_{w}-x_{t}\right)$. Noting that $\prod_{\substack{w<t \\ w, t \neq u}}\left(x_{w}^{2}-x_{t}^{2}\right)$ is the Vandermonde determinant of the $x_{v}^{2}, v \neq u$, it follows that

$$
I_{N}^{(d)}=\frac{1}{\prod_{w<t}\left(x_{w}+x_{t}\right)} \frac{1}{\Delta} \operatorname{det}\left(\begin{array}{cccc}
x_{1}^{2(N-1)} & x_{2}^{2(N-1)} & \cdots & x_{N}^{2(N-1)} \\
x_{1}^{2(N-2)} & x_{2}^{2(N-2)} & \cdots & x_{N}^{2(N-2)} \\
\vdots & \vdots & \ddots & \vdots \\
x_{1}^{4} & x_{2}^{4} & \cdots & x_{N}^{4} \\
x_{1}^{2} & x_{2}^{2} & \cdots & x_{N}^{2} \\
x_{1}^{d} & x_{2}^{d} & \cdots & x_{N}^{d}
\end{array}\right) .
$$

Now recall that for any $N$-tuple $\lambda=\left(\lambda_{1}, \ldots, \lambda_{N}\right)$, the quotient

$$
S_{\lambda}\left(x_{1}, \ldots, x_{N}\right)=\frac{1}{\Delta} \operatorname{det}\left[\left(x_{v}^{\lambda_{u}+N-u}\right)_{u, v=1 \ldots N}\right]
$$

is a polynomial which in the case of a partition (i.e.where $\lambda_{u} \geq \lambda_{u+1}$ ) is called the Schur polynomial associated with $\lambda$ [26]. For $d=2,4, \ldots, 2 N-2$, two rows of the determinant in (B.4) coincide, so $I_{N}^{(d)}=0$. In all other cases, after taking out a factor of $\left(\prod_{u} x_{u}\right)^{\min (2, d)}$ from the determinant and reordering the rows, one obtains a Schur polynomial. For example, for $d<0$, the result is

$$
I_{N}^{(d)}=\frac{\left(\prod_{u} x_{u}^{d}\right) S_{\lambda}}{\prod_{u<v}\left(x_{u}+x_{v}\right)}
$$

with $\lambda=(N-1-d, N-2-d, \ldots, 1-d, 0)$. Among other things, this implies that $I_{N}^{(d)}$ is not, as it may appear from the definition, singular at $x_{u}=x_{v}$.

An important property of $I_{N}^{(d)}$ that we shall need later is its value when all arguments are set equal to unity. The Schur polynomials are the characters of the irreducible representations of $S U(N)$, and their values at $x=1$ are given by the dimension formula

$$
S_{\lambda}(1,1, \ldots, 1)=\prod_{\substack{u, v=1 \ldots N \\ u<v}} \frac{\lambda_{u}-\lambda_{v}+v-u}{v-u} .
$$

For example, for $d=-1$,

$$
S_{(N, N-1, \ldots, 3,2,0)}=2^{(N-1)(N-2) / 2} \frac{(2 N-1) ! !}{(N-1) !}
$$

and therefore

$$
I_{N}^{(-1)}(1,1, \ldots, 1)=2^{1-N} \frac{(2 N-1) ! !}{(N-1) !} \equiv k_{N} .
$$

It is straightforward to compute similarly that for all integer $d$,

$$
I_{N}^{(d)}(1,1, \ldots, 1)=\left(\begin{array}{c}
N-\frac{d}{2}-1 \\
-\frac{d}{2}
\end{array}\right) \equiv \frac{\left(1-\frac{d}{2}\right) \cdots\left((N-1)-\frac{d}{2}\right)}{(N-1) !} .
$$

For later reference note that the large- $N$ limit of this expression (obtained by using Stirling's approximation) has the form

$$
I_{N}^{(d)}(1,1, \ldots, 1)=\frac{\Gamma(N-d / 2)}{\Gamma(1-d / 2) \Gamma(N)}=\frac{N^{-d / 2}}{\Gamma(1-d / 2)}\left(1+O\left(\frac{1}{N}\right)\right) .
$$




\section{B.2 Large- $N$ limit}

We will first consider the simplest non-trivial vacuum configuration, namely, one in which $M_{1}$ of the vacuum expectation values of the scalar fields take one value $\varphi_{u}=a_{1}$ (suppressing the six-dimensional vector index) and the remaining $N-M_{1}=M_{2}$ ones take a second value $\varphi_{u}=a_{2}$, with $M_{1} a_{1}+M_{2} a_{2}=0$. This corresponds to setting $x_{1}=x_{2}=\cdots=x_{M_{1}}=$ $y \equiv\left(\chi-a_{1}\right)^{2}$ and $x_{M_{1}+1}=\cdots=x_{N}=z \equiv\left(\chi-a_{2}\right)^{2}$. Without loss of generality, we take the instanton modulus $\chi$ to be in a region with $y<z$. As a first step, we consider $I_{N}\left(y_{1}, \ldots, y_{M_{1}}, z_{1}, \ldots, z_{M_{2}}\right)$ with non-degenerate arguments satisfying $y_{u}<z_{w}$ and expand

$$
\begin{aligned}
I_{N}\left(y_{1}, \ldots, y_{M_{1}}, z_{1}, \ldots, z_{M_{2}}\right) & =\sum_{u=1 \ldots M_{1}} \frac{1}{y_{u}} \prod_{\substack{v=1 \ldots M_{1} \\
v \neq u}} \frac{y_{v}^{2}}{y_{v}^{2}-y_{u}^{2}} \prod_{w=1 \ldots M_{2}}\left(1-\frac{y_{u}^{2}}{z_{w}^{2}}\right)^{-1} \\
& +\sum_{u=1 \ldots M_{2}} \frac{1}{z_{u}} \prod_{\substack{w=1 \ldots M_{1} \\
w}}\left(1-\left(1-\frac{y_{w}^{2}}{z_{u}^{2}}\right)^{-1}\right) \prod_{\substack{v=1 \ldots M_{2} \\
v \neq u}} \frac{z_{v}^{2}}{z_{v}^{2}-z_{u}^{2}} .
\end{aligned}
$$

The products over $w$ can be rewritten using the complete symmetric functions $h_{r}$,

$$
h_{r}\left(x_{1}, \ldots, x_{n}\right)=\sum_{i_{1}+\cdots+i_{n}=r} x_{1}^{i_{1}} \cdots x_{n}^{i_{n}}, \quad \prod_{i=1}^{n}\left(1-x_{i} t\right)^{-1}=\sum_{r \geq 0} h_{r}\left(x_{1}, \ldots, x_{n}\right) t^{r}
$$

which leads to

$$
\begin{aligned}
& I_{N}\left(y_{1}, \ldots, y_{M_{1}}, z_{1}, \ldots, z_{M_{2}}\right) \\
& =\sum_{r=0}^{\infty} I_{M_{1}}^{(2 r-1)}\left(y_{1}, \ldots, y_{M_{1}}\right) h_{r}\left(z_{1}^{-2}, \ldots, z_{M_{2}}^{-2}\right) \\
& \quad+(-)^{M_{1}} \sum_{r=0}^{\infty} y_{1}^{2} \cdots y_{M_{1}}^{2} h_{r}\left(y_{1}^{2}, \ldots, y_{M_{1}}^{2}\right) I_{M_{2}}^{\left(-2\left(M_{2}+r\right)-1\right)}\left(z_{1}, \ldots, z_{M_{2}}\right) .
\end{aligned}
$$

We can now apply (B.10) and set all $y_{u}=y$ and $z_{u}=z$, giving

$$
\begin{aligned}
& I_{N}(\underbrace{y, \ldots, y}_{M_{1}}, \underbrace{z, \ldots, z}_{M_{2}})=\sum_{r=0}^{\infty}\left(\begin{array}{c}
M_{2}+r-1 \\
r
\end{array}\right)\left(\begin{array}{c}
M_{1}-r-\frac{1}{2} \\
r+\frac{1}{2}
\end{array}\right) y^{2 r-1} z^{-2 r} \\
& \quad+(-)^{M_{1}} \sum_{r=0}^{\infty}\left(\begin{array}{c}
M_{1}+r-1 \\
r
\end{array}\right)\left(\begin{array}{c}
N+r-\frac{1}{2} \\
M_{1}+r+\frac{1}{2}
\end{array}\right) y^{2\left(M_{1}+r\right)} z^{-2\left(M_{1}+r\right)-1} .
\end{aligned}
$$

Now we take the limit $N \rightarrow \infty$ with $m_{1}=M_{1} / N$ and $m_{2}=M_{2} / N$ fixed. The terms in the second line are then negligible. Using the asymptotics (B.11) of the binomial coefficients, we obtain

$$
\begin{gathered}
I_{N}(\underbrace{y, \ldots, y}_{M_{1}}, \underbrace{z, \ldots, z}_{M_{2}}) \stackrel{N \rightarrow \infty}{\longrightarrow} \frac{2}{\sqrt{\pi}} N^{1 / 2} \frac{m_{1}^{1 / 2}}{y} \sum_{r=0}^{\infty}\left(\begin{array}{c}
\frac{1}{2} \\
r
\end{array}\right)\left(\frac{m_{2}}{m_{1}}\right)^{r}\left(\frac{y}{z}\right)^{2 r} \\
=\frac{2}{\sqrt{\pi}}\left(\frac{M_{1}}{\left(\chi-a_{1}\right)^{4}}+\frac{M_{2}}{\left(\chi-a_{2}\right)^{4}}\right)^{1 / 2} .
\end{gathered}
$$


The expression in the square root is the harmonic function $H$ appearing in the two-centre supergravity solution, where the instanton moduli $\chi^{i}$ are identified with the transverse coordinates $y^{i}$ (up to a dimensional scale). The large $N$ limit of the function $I_{N}$ is thus identical, up to a numerical factor, to the classical supergravity volume element $\sqrt{\operatorname{det} g}=$ $\sqrt{H}$. The above analysis straightforwardly generalises to a situation where the vacuum expectation values are degenerate at several centres.

Finally, we consider the behaviour of $I_{N}$ far away from the expectation values, $r \equiv$ $|\chi| \gg \varphi_{u}$. Writing

$$
x_{u}=\left(\chi-\varphi_{u}\right)^{2}=r^{2}\left(1+\varepsilon_{u}\right), \quad \varepsilon_{u}=-2 \frac{\chi \cdot \varphi_{u}}{r^{2}}+\frac{\varphi_{u}^{2}}{r^{2}},
$$

we have $\varepsilon \rightarrow 0$ in that region. From $(\overline{B .6})$ with $d=-1$ we obtain

$$
I_{N}\left(x_{1}, \ldots, x_{N}\right)=\frac{1}{r^{2}} \frac{S_{\lambda}\left(1+\varepsilon_{u}\right)}{\left[\prod_{u}\left(1+\varepsilon_{u}\right)\right]\left[\prod_{u<v}\left(2+\varepsilon_{u}+\varepsilon_{v}\right)\right]} .
$$

The denominator can also be expressed in terms of Schur polynomials [26],

$$
\begin{aligned}
\prod_{u}\left(1+\varepsilon_{u}\right) & =\sum_{r=0}^{n} S_{\left(1^{r}\right)}\left(\varepsilon_{u}\right) \\
\prod_{u<v}\left(2+\varepsilon_{u}+\varepsilon_{v}\right) & =S_{\delta}\left(1+\varepsilon_{u}\right),
\end{aligned}
$$

where the symbol $\left(1^{r}\right)$ denotes the partition $(1, \ldots, 1)$ of $r$, and $\delta=(N-1, N-2, \ldots, 1)$. A formula by A. Lascoux [26, 27] provides an explicit Taylor expansion of arbitrary Schur polynomials around $x_{u}=1$,

$$
S_{\lambda}\left(1+\varepsilon_{1}, \ldots, 1+\varepsilon_{N}\right)=\sum_{\mu \subset \lambda} d_{\lambda \mu} S_{\mu}\left(\varepsilon_{1}, \ldots, \varepsilon_{N}\right),
$$

where the sum is over all partitions $\mu$ with $\mu_{u} \leq \lambda_{u}$, all $u$, and the coefficients $d_{\lambda \mu}$ are given by

$$
d_{\lambda \mu}=\operatorname{det}\left[\left(\begin{array}{c}
\lambda_{u}+N-u \\
\mu_{v}+N-v
\end{array}\right)\right]_{u, v=1, \ldots, N} .
$$

With the help of Mathematica, it is now possible to compute an expansion of $I_{N}\left(1+\varepsilon_{u}\right)$ in terms of Schur polynomials of the $\varepsilon_{u}$,

$$
I_{N}\left(1+\varepsilon_{u}\right)=\underbrace{\frac{d_{\lambda 0}}{d_{\delta 0}}}_{k_{N}} \frac{1}{r^{2}}\left(1+\frac{1}{N} S_{(1)}+\frac{3 N+1}{2 N(N+1)}\left(S_{(2)}-S_{(1,1)}\right)+\cdots\right)
$$

In order to compare with the supergravity calculation it turns out to be more useful to rewrite this expansion [26] in terms of power sums, $p_{k}=p_{k}(\varepsilon)=\sum_{u=1}^{N} \varepsilon_{u}^{k}$,

$$
\begin{aligned}
I_{N}\left(x_{1}, \ldots, x_{N}\right) & =\frac{k_{N}}{r^{2}}\left[1-\frac{p_{1}}{N}+\frac{(3 N+2) p_{2}-p_{1}^{2}}{2 N(N+1)}\right. \\
& \left.-\frac{\left(4 N^{2}+9 N+4\right) p_{3}-3(N+1) p_{2} p_{1}+p_{1}^{3}}{2 N(N+1)(N+2)}+O\left(\varepsilon^{4}\right)\right] .
\end{aligned}
$$


with the large- $N$ limit

$$
\begin{aligned}
I_{N}\left(x_{1}, \ldots, x_{N}\right) \stackrel{N \rightarrow \infty}{\longrightarrow} \frac{1}{r^{2}} \frac{2 \sqrt{N}}{\sqrt{\pi}} & {\left[1-\frac{p_{1}}{N}+\frac{3 N p_{2}-p_{1}^{2}}{2 N^{2}}\right.} \\
& \left.-\frac{4 N^{2} p_{3}-3 N p_{2} p_{1}+p_{1}^{3}}{2 N^{3}}+O\left(\varepsilon^{4}\right)\right] .
\end{aligned}
$$

On the supergravity side, the multi-centred harmonic function with arbitrary locations $\varphi_{u}$ of the centres has the following large-distance expansion,

$$
\begin{aligned}
\sqrt{H} & =\left[\sum_{u=1}^{N} \frac{1}{\left(\chi-\varphi_{u}\right)^{4}}\right]^{1 / 2}=\frac{1}{r^{2}}\left[\sum_{u=1}^{N} \frac{1}{\left(1+\varepsilon_{u}\right)^{2}}\right]^{1 / 2} \\
& =\frac{\sqrt{N}}{r^{2}}\left[1-\frac{p_{1}}{N}+\frac{3 N p_{2}-p_{1}^{2}}{2 N^{2}}-\frac{4 N^{2} p_{3}-3 N p_{2} p_{1}+p_{1}^{3}}{2 N^{3}}+O\left(\varepsilon^{4}\right)\right] .
\end{aligned}
$$

This again coincides with the large- $N$ limit (B.25) of $I_{N}$. We have checked this equality up to eighth order in $\varepsilon$ so that

$$
I_{N}\left(x_{1}, \ldots, x_{N}\right) \stackrel{N \rightarrow \infty}{\longrightarrow} \frac{2}{\sqrt{\pi}} \sqrt{H}+r^{-2} O\left(\left(\frac{\varphi_{u}}{r}\right)^{9}\right) .
$$

\section{References}

[1] J. Maldacena, The large $N$ limit of superconformal field theories and supergravity, Adv. Theor. Math. Phys. 2 (1998) 231, Int. J. Theor. Phys. 38 (1999) 1113, hep-th/9711200.

[2] S. S. Gubser, I. R. Klebanov and A. M. Polyakov, Gauge theory correlators from non-critical string theory, Phys. Lett. B 428 (1998) 105, hep-th/9802109.

[3] E. Witten, Anti de Sitter space and holography, Adv. Theor. Math. Phys. 2 (1998) 253, hep-th/9802150.

[4] T. Banks and M. B. Green, Nonperturbative effects in $A d S_{5} \times S^{5}$ string theory and $D=4$ SUSY Yang-Mills, J. High Energy Phys. 05 (1998) 002, hep-th/9804170.

[5] M. Bianchi, M. B. Green, S. Kovacs and G. C. Rossi, Instantons in supersymmetric Yang-Mills and D-instantons in IIB superstring theory, J. High Energy Phys. 08 (1998) 013, hep-th/9807033.

[6] N. Dorey, T. J. Hollowood, V. V. Khoze, M. P. Mattis and S. Vandoren, Multi-instanton calculus and the AdS/CFT correspondence in $N=4$ superconformal field theory, Nucl. Phys. B 552 (1999) 88, hep-th/9901128.

[7] M. B. Green and S. Kovacs, Instanton-induced Yang-Mills correlation functions at large $N$ and their $A d S_{5} \times S^{5}$ duals, hep-th/0212332.

[8] P. Kraus, F. Larsen and S. P. Trivedi, The Coulomb branch of gauge theories from rotating branes, J. High Energy Phys. 03 (1999) 003, hep-th/9811120.

[9] I. R. Klebanov and E. Witten, AdS/CFT correspondence and symmetry breaking, Nucl. Phys. B 556 (1999) 89, hep-th/9905104.

[10] D. Z. Freedman, S. S. Gubser, K. Pilch and N. P. Warner, Continuous distributions of D3-branes and gauged supergravity, J. High Energy Phys. 07 (2000) 038, hep-th/9906194. 
[11] A. Brandhuber and K. Sfetsos, Wilson loops from multicentre and rotating branes, mass gaps and phase structure in gauge theories, Adv. Theor. Math. Phys. 3 (1999) 851, hep-th/9906201.

[12] N. Dorey, T. J. Hollowood and V. V. Khoze, The D-instanton partition function, J. High Energy Phys. 03 (2001) 040, hep-th/0011247.

[13] G. 't Hooft, Computation of the quantum effects due to a four-dimensional pseudoparticle, Phys. Rev. D 14 (1976) 3432.

[14] I. Affleck, On constrained instantons, Nucl. Phys. B 191 (1981) 429.

[15] N. Dorey, T. J. Hollowood, V. V. Khoze, M. P. Mattis, The Calculus of Many Instantons, Phys. Rept. 371 (2002) 231.

[16] M. B. Green and M. Gutperle, Effects of D-instantons, Nucl. Phys. B 498 (1997) 195, hep-th/9701093.

[17] M. B. Green, M. Gutperle, P. Vanhove, One loop in eleven dimensions, Phys. Lett. B 409 (1997) 177, hep-th/9706175.

[18] M. B. Green and S. Sethi, Supersymmetry constraints on type IIB supergravity, Phys. Rev. D 59 (1999) 046006, hep-th/9808061.

[19] J. H. Schwarz, Covariant field equations of chiral $N=2 D=10$ supergravity, Nucl. Phys. B 226 (1983) 269.

[20] P. Howe and P. West, The complete $N=2, D=10$ supergravity, Nucl. Phys. B 238 (1984) 181 .

[21] S. de Haro, A. Sinkovics and K. Skenderis, On a supersymmetric completion of the $R^{4}$ term in IIB supergravity, Phys. Rev. D 67 (2003) 084010, hep-th/0210080.

[22] K. Peeters, P. Vanhove and A. Westerberg, Chiral splitting and world-sheet gravitinos in higher-derivative string amplitudes, Class. and Quant. Grav. 19 (2002) 2699, hep-th/0112157.

[23] K. Peeters and A. Westerberg, The Ramond-Ramond sector of string theory beyond leading order, hep-th/0307298.

[24] M. J. Duff and J. X. Lu, The selfdual type IIB superthreebrane, Phys. Lett. B 273 (1991) 409.

[25] G. W. Gibbons, M. B. Green and M. J. Perry, Instantons and seven-branes in type IIB superstring theory, Phys. Lett. B 370 (1996) 37, hep-th/9511080.

[26] I. G. Macdonald, Symmetric Functions and Hall Polynomials, Clarendon Press, 1979.

[27] A. Lascoux, Classes de Chern d'un produit tensoriel, C. R. Acad. Sci., Paris, Sér. A 286, 385-387 (1978). 